Nonlinear effects of retinal amacrine cells

\title{
Diverse nonlinear modulation of visual features by retinal amacrine cells
}

\author{
Neda Nategh ${ }^{1,3}$, Mihai Manu ${ }^{2,4}$ and Stephen A. Baccus ${ }^{2, *}$ \\ ${ }^{1}$ Department of Electrical Engineering, Stanford University, Stanford, CA 94305 \\ ${ }^{2}$ Department of Neurobiology, Stanford University School of Medicine, Stanford, CA, USA. \\ ${ }^{3}$ Present address: Department of Electrical \& Computer Engineering, University of Utah, Salt \\ Lake City, UT 84112 \\ ${ }^{4}$ Department of Neurosurgery, Cologne-Merheim Medical Center, Witten/Herdecke University \\ School of Medicine, Germany \\ *Correspondence and Lead Contact: baccus@stanford.edu
}

\section{SUMMARY}

Inhibitory interneurons are diverse in the nervous system, though most have unknown functions. In sensory systems, two broad classes of computation have been considered linear effects that generate the classical receptive field and nonlinear modulation that mediates non-classical contextual effects. Here we analyze salamander retinal amacrine cells using a general approach to directly measure and model how an interneuron pathway influences computation. Using simultaneous intracellular and multielectrode recording, we measure the linear feature contributed by an amacrine pathway and nonlinear modulatory effects on other visual features. We find great diversity in the functional effects of amacrine cells, with even apparently simple, linear amacrine cells creating both linear and diverse modulatory effects such as divisive gain control, polarity reversal and shifting threshold on distinct visual features conveyed to single target ganglion cells. The functional effects of amacrine cells parallel the previously noted anatomical and molecular heterogeneity of this interneuron population.

\section{Highlights}

- Some amacrine cells contribute a linear feature to ganglion cells but others do not

- The visual feature conveyed by an amacrine pathway improves a ganglion cell model

- One amacrine-ganglion cell pair has different modulatory effects on different features

- Amacrine cells with linear responses have highly diverse nonlinear modulary effects 
Nonlinear effects of retinal amacrine cells

\section{INTRODUCTION}

Sensory neurons combine signals delivered through multiple parallel pathways to produce computations in neural circuits. Although there have been many measurements of sensory responses in particular neurons, there has been limited understanding about how parallel pathways interact and what their functional implications are. This is particularly true for interactions between inhibitory interneurons and excitatory pathways, where the diversity of connections, cell-types and structure makes it difficult to understand how signals in separate pathways interact (Baccus, 2007; Markram et al., 2004; Masland, 2012b; Mott and Dingledine, 2003; Roska et al., 2006).

Historically, understanding the role of interneurons' functions has been a challenge, mainly due to the limitations in selectively perturbing neurons in real time and recording the effects of such perturbations. As a result, many of the reported roles of inhibitory circuits are based on correlative evidence, supported by a variety of computational models (Isaacson and Scanziani, 2011; Jadzinsky and Baccus, 2013).

Yet despite many new methods to perturb interneurons selectively and record from neural populations, these techniques have not been applied in a general approach to understand how interneurons produce computations in a circuit, in particular because of the lack of computational methods fit directly to physiological data, to understand their effects quantitatively. The lack of an accepted way to understand complex nonlinear interactions in the nervous system is a widespread limitation that can potentially lead to misinterpretations of experimental perturbations of the nervous system (Otchy et al., 2015).

Retinal amacrine cells are a class of diverse inhibitory interneurons that participate in multiple forms of inhibitory interactions including feedback, feedforward and lateral inhibition, 
Nonlinear effects of retinal amacrine cells

targeting bipolar cells, ganglion cells, and other amacrine cells, and are mostly diversified according to the types of ganglion cells (Masland, 2012a; Sanes and Masland, 2015; Werblin, 2011). Methods exist to present visual stimuli while perturbing single interneurons and recording the effects with a multielectrode array (Asari and Meister, 2012, 2014; Baccus et al., 2008; de Vries et al., 2011; Manu and Baccus, 2011). Finally, the precision to which the retina responds (Berry et al., 1997), makes it possible to connect neuron-level mechanisms with circuit-level functions as represented by a computational model that accurately fits the neuron's response.

When considering the functional contribution of an interneuron pathway, one can divide signals into two broad components, those flowing through the interneuron, and those traveling through pathways parallel to the interneuron to the output cell. The interaction between those two pathways have been divided into two classes, a linear or classical effect (Dong and Hare, 2003; Hubel and Wiesel, 1962; Machens et al., 2004; Masland et al., 1984; Molnar et al., 2009; van Hateren, 2005), whereby a neural pathway contributes to the linear or classical receptive field, and a modulatory effect whereby a neural pathway interacts nonlinearly with the output of other parallel pathways (Carvalho and Buonomano, 2009; Chance et al., 2002; Chen et al., 2010; Hallum and Movshon, 2014; Katzner et al., 2011; Mitchell and Silver, 2003; Molnar and Werblin, 2007; Olsen and Wilson, 2008; Ölveczky et al., 2003; Roska and Werblin, 2003). There has not existed an approach to study the overall contribution of specific neurons to these two types of effects.

Here we use a general method to directly measure the visual feature transmitted by a neural pathway, and infer signals flowing through all other pathways. Furthermore, we use a general approach to analyze the linear and nonlinear interactions of the signals flowing through an interneuron pathway and other pathways. Using simultaneous intracellular and multielectrode 
Nonlinear effects of retinal amacrine cells

recording in the isolated retina, we found a range of effects from amacrine cells that include both mediating a component of the average visual feature encoded by ganglion cell, and diverse modulatory effects on other neural pathways. The range of modulatory effects represented a continuum that comprised six general categories, including different combinations of divisive gain control, shifting of threshold, an additive inhibitory effect, and reversal of polarity. Furthermore, even amacrine cells with simple, linear responses under stimulation by a uniform visual field could create linear and diverse types of nonlinear effects on multiple different features of single target ganglion cells. These results indicate that individual amacrine cells simultaneously create a wide range modulatory effects on different visual features transmitted by the retina. Any complete description of interneuron function in the retina must account for this diverse modulation.

\section{RESULTS}

\section{The stimulus feature transmitted by an amacrine pathway}

We intracellularly measured and manipulated the activity of individual amacrine cells including sustained $(\mathrm{n}=8)$ and transient $(\mathrm{n}=3)$ in the isolated salamander retina and simultaneously recorded the spiking activity of many ganglion cells $(n=153)$ at once in response to visual stimulation and amacrine perturbation using an array of extracellular electrodes (Fig. 1).

We first analyzed the visual feature transmitted by the amacrine pathway, which is formed in two stages - the amacrine cell's visual response, and its transmission. We measured the visual response of amacrine cells by fitting a linear-nonlinear (LN) model consisting of a linear temporal filter followed by a time-dependent, or static nonlinearity (Fig. 2A). The linear filter represents the average effect of a brief flash of light on the amacrine cell membrane potential, or 
Nonlinear effects of retinal amacrine cells

equivalently can be interpreted as the time-reverse of the visual stimulus to which the amacrine cell was most sensitive on average.

To characterize the second stage of the interneuron pathway - how signals transmitted through individual amacrine cells contributed to the ganglion cell response - we injected white noise current into the amacrine cell while presenting a white noise visual stimulus. Because the inner retina adapts to the stimulus contrast by changing its sensitivity (Baccus and Meister, 2002; Kim and Rieke, 2001), the visual stimulus was included to maintain the retina in a similar state of adaptation in the current injection and control conditions. We then computed an LN model between the amacrine current and the ganglion cell spike train (Manu and Baccus, 2011) (see Experimental procedures, Fig. 2B). In this transmission model, the transmission filter represents the average effect of current on the ganglion cell firing rate. The linear transmission filters were mostly inhibitory with different timecourses (Fig. 2C). Effects were seen on only a subtype of ganglion cells (Fig. 2 E, F). Consistent with previous results from steady current pulses (de Vries et al., 2011), transmission dynamics in some cases were specific to the amacrine cell - ganglion cell pair, with single amacrine cells having different dynamics for different target ganglion cells (Supp. Fig. S1).

Because the input signal to this cascade has a Gaussian probability distribution, the overall linear feature conveyed by this pathway can be obtained by convolving the two linear filters whether the amacrine visual response is linear or nonlinear (Hunter and Korenberg, 1986). In combining these two filters, we avoided a double contribution from the membrane time constant by deconvolving by an exponential filter representing that time constant (Manu and Baccus, 2011). The features conveyed by the amacrine pathway were typically monophasic and positive, 
Nonlinear effects of retinal amacrine cells

formed by a negative response to light (Off response) followed by an inhibitory transmission; there was diversity in the time course of the feature (Fig. 2D).

\section{Signals traveling through parallel neural pathways}

To identify the signals conveyed by neural pathways other than through the amacrine cell, we took the amacrine pathway contribution out of the $n$-dimensional stimulus by computing the (n-1) dimensional stimulus space orthogonal to the amacrine feature (See Experimental Procedures). Within this new stimulus subspace, we computed the ganglion cell's spike-triggered average (STA) feature, the orthogonal STA (oSTA) (Fig. 3A). This feature represents the average of other visual features encoded by the ganglion cell, that were not conveyed by (were orthogonal to) the amacrine pathway. This two-pathway model thus encodes two features of the stimulus, one average feature encoded by the amacrine cell, and one average feature encoded by all other pathways. Note that this does not account for the possibility that other interneuron pathways may be conveying the same feature as the amacrine cell, and so in this model we effectively refer all of the amacrine feature to be encoded by the amacrine pathway.

Comparing the full STA of the ganglion cell and the oSTA, we found that in some cases the two differed, meaning that the amacrine pathway contributed to the average visual feature conveyed by the ganglion cell (Fig. 3B). In other cases, however, the STA and oSTA were nearly identical, meaning that the amacrine pathway feature did not contribute to the linear receptive field of the ganglion cell (Fig. 3C). The range of differences between the STA and orthogonal STA indicated substantial diversity in the linear contribution of an amacrine cell to a ganglion cell's response (Fig. 3D). 
Nonlinear effects of retinal amacrine cells

Amacrine cells that contributed to the ganglion cell STA had a smaller time-to-peak difference between the amacrine cells' own STA and the ganglion cell STA. For other properties such as ganglion cell STA, the timing of the amacrine transmission filter and the overall feature conveyed by the amacrine cell pathway, amacrine cells that contributed to the ganglion cell STA were similar to those that did not (Fig. 3E). Thus the contribution of an amacrine cell to the average ganglion cell visual feature did not appear to be a property of either cell type, but instead depended on the relative timing of the amacrine and ganglion cell responses.

\section{A model using the amacrine cell pathway more accurately predicts the ganglion cell}

\section{response}

To examine if the amacrine pathway feature was necessary to capture the ganglion cell's firing rate modulation in response to a visual stimulus, we compared whether a model consisting of the amacrine pathway and an orthogonal pathway could predict the ganglion cell more accurately than a single pathway LN model. The two pathway model combined the two pathways with a two-dimensional nonlinearity that mapped the output of the two pathways to the ganglion cell firing rate. We computed the model using the ganglion cell and amacrine cell response, and tested the model on a separate section of data, which was held out from the data used to fit the model. Figure 4 shows the firing rate of a sample ganglion cell using only the STA feature (an LN model), or a two-pathway model using the amacrine feature and average orthogonal feature. A third case was tested using a two pathway model fit using the ganglion cell response alone using the standard method of spike-triggered covariance (STC) analysis (Schwartz et al., 2006; Simoncelli et al., 2004). The accuracy of the amacrine model exceeded that of the LN model, and was similar to the two-pathway STC model. This increased 
Nonlinear effects of retinal amacrine cells

performance was not simply because there were more parameters in the multi-pathway models, because all results were cross-validated on different data than were used to fit the model, and in fact a model with more parameters would be expected to perform worse on a different test set of data due to overfitting of the training data set. Note that the standard STC analysis provides no information as to stimulus features conveyed by particular interneuron pathways. Thus, measuring a stimulus feature directly from an interneuron pathway creates a model that is both more accurate and represents a verified internal computation in the circuit.

\section{A general analysis of modulation in amacrine pathways}

A ganglion cell receives input from multiple neural pathways, and each pathway conveys its own preferred stimulus feature. An interneuron could potentially affect each of these features differently. We were interested in understanding (1) what ganglion cell visual features are affected by the amacrine pathway? (2) what kind of nonlinear interaction exists between amacrine pathway and other input pathways to a ganglion cell? and (3) are the amacrine effects different for different features? To address these questions, we decomposed the stimulus orthogonal to the amacrine feature into its principal components, each representing a separate feature, with all of these features being orthogonal to the amacrine feature. We used the standard approach of spike triggered covariance (STC) analysis to recover the entire linear subspace representing the space spanned by the features encoded by the multiple pathways feeding into a ganglion cell that does not include the amacrine pathway feature. For each ganglion cell, we recovered up to several features that were orthogonal to the amacrine feature, which do not necessarily correspond to other specific neural pathways (Fig.5C). 
Nonlinear effects of retinal amacrine cells

To quantify how the amacrine feature was combined with each of these orthogonal features, we computed for each feature a two-dimensional nonlinear function whose inputs were the projection of stimulus on the amacrine pathway feature, and the projection of the stimulus on that orthogonal ganglion cell feature (Fig. 5A and B). The output of this two-dimensional nonlinearity was the ganglion cell firing rate. Thus, this two-dimensional nonlinearity represented the firing rate as a function of the similarity of the stimulus and the ganglion cell visual feature, for different levels of the amacrine pathway output (Fig. 5D). Several different categories of effects were observed in these nonlinearities. In the example shown in Fig. 5D, one effect observed was that as an amacrine cell changed from depolarized to hyperpolarized, the ganglion cell response changed with respect to light from Off-type to On-type (Fig. 5D, first column). Previously it has been shown that the ganglion cell light response can change sign due to peripheral visual stimulation (Geffen et al., 2007). Our current analysis shows that a local amacrine pathway can change the sign of a ganglion cell's response to light on a fast time scale. This result illustrates how inhibitory interneurons can rapidly and dramatically alter the behavior of the output neurons in the course of a computation.

For a different orthogonal stimulus feature of the same amacrine-ganglion cell pair, the same analysis revealed a different type of nonlinear effect of amacrine transmission, an additive effect on firing, such that the baseline level was high when the amacrine cell was hyperpolarized (Fig 5D, col. 2). For the third ganglion cell's significant feature, the cell was suppressed when the stimulus was similar or opposite in sign to this ganglion cell feature (Fig 5D, col. 3). Firing, however was also suppressed by the amacrine feature. For the fourth significant ganglion cell's dimension, the amacrine feature caused a divisive depression (Fig. 5D, col. 4). Note also that even though the amacrine effect on the firing rate is similar for all of these features, in the sense 
Nonlinear effects of retinal amacrine cells

that whenever amacrine cell is hyperpolarized there is greater firing, the nature of the interaction of the amacrine output with the ganglion cell preferred feature is very different. This analysis reveals that simply designating a cell as inhibitory is insufficient to account for the complexity of its effects. Furthermore, even in a single amacrine-ganglion cell pair multiple types of effects can occur.

We could identify six different categories for the nonlinear modulatory effect of the amacrine pathway on the ganglion cell's light response, which included changing the sensitivity, controlling the gain, changing in the threshold, changing both gain and threshold, an additive inhibitory effect, and reversal of polarity, or alternating between Off- and On-type ganglion cell (Fig. 6). Figure 6A-C shows how these categories correspond to a shifting and scaling of the onedimensional nonlinear functions across different polarizations of the amacrine cell. As a strong threshold exists at the bipolar cell terminal, a horizontal shifting and scaling could potentially correspond to presynaptic inhibition, and a vertical shifting or scaling could potentially correspond to postsynaptic inhibition, although we did not specfically test these mechanistic hypotheses here. The effects found for the pairs studied here were not specific for a particular amacrine cell or ganglion cell type, but were specific for each amacrine-ganglion cell's feature pair, with up to eight different features of a single cell pair with different effects (Fig. 6D).

\section{Diverse modulatory effects on the ganglion cell population}

To further analyze the modulatory effects of amacrine cells across the population of cell pairs, we parameterized the two-dimensional nonlinearity for each amacrine - ganglion cell feature pair, measured directly from ganglion spiking response, with a set of quadratic logistic functions as described in equation (10) (see Experimental Procedures for details). These 
Nonlinear effects of retinal amacrine cells

functions are consistent with a so-called maximum noise entropy model, that has the highest uncertainty about the neuron's response, and so are least constrained or biased with respect to a given set of measurements (Jaynes, 1957a, b). This model-based description was necessary as the modulations caused by the amacrine cell's signal operated on the same time scale as the response itself. The resulting functional characterization then enabled the separation between the mechanisms underlying the amacrine fast modulations and those that give rise to the stimulusevoked response.

For the ganglion cell's response at different levels of amacrine polarization, we fit a function with only three parameters to different one-dimensional response nonlinearities corresponding to each of these levels and saw how the amacrine cell changes these parameters (Fig. 7A, see Experimental Procedures). We fit the ganglion cell nonlinearity and one level of amacrine output to the equation,

$$
P\left(r_{i}=1 \mid g_{i}\right)=\frac{1}{1+\exp \left[\alpha+\beta\left(g_{i}-\gamma\right)^{2}\right]}
$$

where $P\left(r_{i}=1 \mid g_{i}\right)$ is the probability that the response is a spike given a particular value of $g_{i}$, which is the projection of the stimulus on one of the ganglion cell's orthogonal feature dimension at time $i$. The estimated parameters $\alpha, \beta, \gamma$ change depending on the output of the amacrine pathway, which in turn depends on the specific stimulus sequence. Thus the parameters, $\alpha, \beta, \gamma$ are controlled by the amacrine pathway, and describe the immediate ganglion cell visual response to light. These parameters are not varied independently and so can only be jointly interpreted. Roughly speaking, $\gamma$ mostly takes care of the lateral shift or threshold, $\beta$ mostly takes care of the sensitivity, $\alpha$ takes care of mostly the vertical scaling and the amplitude, and additive 
Nonlinear effects of retinal amacrine cells

shift is controlled by the combination of all these parameters together. Polarity reversal can be realized by changing the sign of $\beta$.

Figure 7B-D shows the pairwise distribution of these parameters for 101 pairs of amacrine and significant ganglion cell's orthogonal features out of total of 160 pairs, selected by additional goodness of fit criteria, as well as how the parameters change as a function of amacrine pathway output. Although the nonlinear effects for different feature pairs did not appear to cluster, there were regions of varying density that are suggestive of more common types of effects (Fig. 7 BC). We observed substantial diversity in the types of nonlinear effects of amacrine cells. Additionally, the lack of clustering based on the type of either amacrine cells or ganglion cells indicates a continuum of possible behaviors. Furthermore, amacrine cells can have multiple effects on different features of the same ganglion cell. To validate the precision of the estimates of the effect for each feature pair, we performed a cross-validation procedure for the parameters estimation to find the standard error in estimation of each parameter (see Experimental Procedures). The results indicate that the error bars associated with each estimated parameter are smaller than variations across data points, confirming that many different behaviors are observed that form a continuum of distinct effects (Fig. 7C). Thus, amacrine cells cause diverse and specific modulatory effects on multiple stimulus features, even within single target ganglion cells.

\section{DISCUSSION}

Using our experimental and computational approach, we observed considerable diversity in the functional effects of amacrine cells. The first of these effects involved the linear contribution in which amacrine pathway contributes in building the receptive field of the output neuron. Such effects show that the amacrine cell contributes directly to the average ganglion cell stimulus 
Nonlinear effects of retinal amacrine cells

selectivity. Secondly, by measuring the nonlinear effects of the amacrine cells, we saw great diversity in modulatory effects of these inhibitory interneurons on the response of ganglion cells. Amacrine cells can shift the threshold of the ganglion cell's response function, or control the ganglion cell's response sensitivity, or suppress a feature encoded by the ganglion cell, or gate the flow of information i.e. ganglion cell's polarity reversal. Moreover, we saw diversity in how these effects are combined, with different effects combined for a single ganglion cell feature, and different effects for different features even with the same amacrine-ganglion cell pairs. These effects are modulatory, meaning that one feature - the amacrine feature - changes the response to other features. Across many amacrine cells with diverse effects, the amacrine cell population defines a context that modulates multiple other features conveyed by the ganglion cell.

\section{Linking a Functional Pathway to a Neural Pathway}

An important question in the study of sensory processing neurons is that what stimulus features drive the spiking response of the neurons. Recent studies have shown that many neurons respond to more than one feature in a high-dimensional stimulus space (Atencio et al., 2008; Brenner et al., 2000b; Cantrell et al., 2010; Chen et al., 2007; Fairhall et al., 2006; Felsen et al., 2005; Fox et al., 2010; Hong et al., 2007; Horwitz et al., 2005, 2007; Kim et al., 2010; Maravall et al., 2007; Rust et al., 2005; Sincich et al., 2009; Tanabe et al., 2011; Touryan et al., 2005; Touryan et al., 2002). Although dimensionality reduction approaches, such as spike-triggered covariance (STC) and its extensions (Bialek and van Steveninck, 2005; Paninski, 2003; Park and Pillow, 2011; Pillow and Simoncelli, 2006; Schwartz et al., 2002; Schwartz et al., 2006; Van Steveninck and Bialek, 1988) or maximally informative dimensions (MID) and its extensions (Fitzgerald et al., 2011; Rajan and Bialek, 2013; Sharpee et al., 2004), have been successful to 
Nonlinear effects of retinal amacrine cells

find a relevant linear subspace that causes a change in the neuron's response, the resulting features can not be easily interpreted in terms of functional computations. As a result, this subspace description provides little insight into the neural circuitry that gives rise to the neuron's response. Moreover, it is not clear that dimensions extracted by these methods relate to neural pathways, and we only know that the space of these extracted dimensions encompasses the space of features encoded by all the neural pathways feeding into one neuron. Although other work has tried to rank these stimulus dimensions according to how much information they capture (Pillow and Simoncelli, 2006), it is still difficult to link the resulting features to the neural pathways encoding those features.

In this study, by actually measuring the amacrine pathway feature, we can assign a neural identity to one of those pathways and characterize its effect in a way that would not be possible by modeling based on the ganglion cell response alone.

\section{Implications of amacrine cell modulation for natural scene processing}

It has been suggested that neural representations of stimuli may be optimized in some way to the statistics of the natural environment (Attneave, 1954; Barlow, 2001; Barlow, 1961; Twer and MacLeod, 2001). An important property of natural signals is redundancy, because one spatial or temporal portion of a natural scene may be predicted from other areas nearby or signals from the past (Dong and Atick, 1995; Field, 1987). It has often been suggested that sensory systems are adapted to the statistics of the signals they encounter, and the underlying principle for this adaptation is to provide a statistically efficient representation of the information in the environment (Attneave, 1954). In the context of neural sensory processing this efficient coding hypothesis suggests that the common role of any early sensory system is to remove this statistical 
Nonlinear effects of retinal amacrine cells

redundancy to provide an independent neural representation of the natural signals (Barlow, 1961; Simoncelli and Olshausen, 2001). Initial efforts to support this theory have derived an optimal set of linear basis functions such that the redundancy in representation of a natural signal is minimized. These basis functions have been shown to have properties similar to receptive fields in the visual cortex (Bell and Sejnowski, 1997; Olshausen, 1996; van Hateren and van der Schaaf, 1998). It has also been shown that the optimal spatial filter for a simplified model of the retina resembles the center-surround receptive fields of retinal ganglion cells (Atick and Redlich, 1990, 1993).

However, the statistics of the natural signals are too complex to be optimally decomposed by only a linear transformation. Moreover, the neural responses are highly nonlinear, for example the necessity of rectification or saturation of responses due to the physiological constraints (Carandini et al., 1997; DeAngelis et al., 1992; Javel et al., 1978; Kapadia et al., 1999; Knierim and Van Essen, 1992; Levitt and Lund, 1997; Sceniak et al., 1999; Shapley and Enroth-Cugell, 1984; Slllito et al., 1995). Recent studies in primary visual cortex V1 and in the retina have shown that nonlinear relationships between stimulus and neuron's response play an important role in decorrelating the visual image and therefore in the efficient coding of natural signals (Pitkow and Meister, 2012; Schwartz and Simoncelli, 2001).

A class of multidimensional nonlinear models that has been able to link the natural signal statistics to the neural response properties exhibits so-called divisive normalization. The intuition behind this model is to divide the squared signal by its variance as predicted from the linear combination of its neighbors to obtain a representation that is approximately univariate. A divisive normalization describes the firing rate responses of cortical neurons as the ratio between the output of a linear receptive field and the magnitude of a set of different visual features 
Nonlinear effects of retinal amacrine cells

(Heeger, 1992). Such models are consistent with responses found across cortical areas and sensory modalities (Carandini and Heeger, 2012). The important functional role of divisive normalization, from a principled scene-statistics perspective, is the efficient encoding of natural signal by implementing redundancy reduction (Coen-Cagli et al., 2009; Karklin and Lewicki, 2005; Schwartz and Simoncelli, 2001; Spratling, 2012). If different visual features often occur together, in other words they are redundant, then if one feature suppresses the other it will tend to create a less redundant representation.

The amacrine pathways we have studied induce predominantly scaling and shifting effects. These effects are consistent with a key property of divisive normalization, namely that visual features reduce the sensitivity of other visual features. Our findings suggest that the normalizing directions are in fact the features encoded by the amacrine cells, and that the amacrine cell's own preferred or optimal feature can be different from the preferred or optimal feature of the ganglion cell. We propose that a major function of amacrine cell circuits may be to undo the receptive field dependencies of multiple visual pathways by controlling the gain and sensitivity along the features encoded by each of these pathways.

\section{Spatiotemporal studies}

To test this redundancy reduction hypothesis, it will be necessary to measure amacrine nonlinear modulations when the features of amacrine pathway and other ganglion cell's incoming pathways are coincident. Therefore, it will be necessary to repeat our experimental and computational procedure while presenting a spatiotemporal visual stimulus containing the type of structure observed in natural scenes. In our current analysis, because stimuli were composed of a uniform field, we only measured the contribution of the temporal component of the stimulus. 
Nonlinear effects of retinal amacrine cells

However, all amacrine and ganglion cells were within $<200 \mu \mathrm{m}$, and thus are highly likely to have overlapping spatial receptive fields. It is thus likely that amacrine and ganglion cell features will be activated together by stimuli with sharp transitions such as an edge. Nevertheless, it will be important to directly measure the spatiotemporal filters of amacrine and ganglion cell features.

Here we have presented a systematic way to understand the functional contribution of an interneuron. Consistent with the known anatomical diversity of amacrine cells (Masland, 2001), our findings about the linear and nonlinear contributions of the amacrine pathway to retinal output reveal diverse modulatory effects. Diverse interneuron circuits give the retina - and the brain - substantial complexity. Thus, revealing a general function for interneurons by studying a relatively simple circuit like the retina can substantially help toward understanding the organization and function of other inhibitory circuits in the brain, and thereby the neural circuit and mechanism underlying each neural computation.

Acknowledgements: We thank K. Boahen, S. Ganguli and K. Shenoy for helpful discussions. This work was supported by grants from the NEI, Pew Charitable Trusts, McKnight Endowment Fund for Neuroscience, the Alfred P. Sloan Foundation and the E. Matilda Ziegler Foundation (S.A.B.).

Author Contributions. N. N., M. M. and S.A.B. designed the study, M. M. and N. N. performed the experiments and analysis, and N. N. and S.A.B. wrote the manuscript. 
Nonlinear effects of retinal amacrine cells

\section{Figure Legends}

\section{Figure 1. Measuring responses and transmission in an amacrine cell pathway. (A) Diagram}

of experimental setup for simultaneous intracellular and multielectrode array recording: a uniform field randomly flickering visual stimulus with a white noise Gaussian distribution is projected through a video monitor onto an intact isolated salamander retina. Simultaneously, Gaussian white noise current (orange trace) was injected intracellularly into an amacrine cell (current condition) or the amacrine cell membrane voltage (green trace) was recorded intracellularly using a sharp microelectrode (control condition). A multi-electrode array of about 60 extracellular electrodes recorded spiking activities of multiple ganglion cells simultaneously. Multiple traces on the left represent spiking response of multiple ganglion cells over time to a single stimulus trial during control condition (orange spikes) and during current condition (green spikes). (B) The membrane potential response of a sustained Off-type amacrine cell (top row), the response of a transient On-Off type amacrine cell (middle row), to a uniform field flashing stimulus (bottom row). (C) (Top) Receptive fields of a single amacrine cell and multiple ganglion cells. Each oval indicates one standard deviation of a two-dimensional Gaussian fit to the receptive field mapped using a white noise checkerboard stimulus. Red oval indicates an amacrine cell receptive field; black ovals indicate ganglion cells affected by current injection into the amacrine cell. Grey ovals are unaffected ganglion cells. (Bottom) Temporal filters computed for recorded cells, including: amacrine visual filter (red), computing by correlating a uniform-field visual stimulus and the amacrine cell membrane potential; ganglion cell visual filters, computed by correlating the visual stimulus and ganglion spikes for the affected ganglion cells (black) and all other recorded ganglion cells (grey); and an amacrine transmission filter, 
Nonlinear effects of retinal amacrine cells

between the amacrine cell and one affected ganglion cell (blue) computed by correlating a white noise current stimulus injected into the amacrine cell and the ganglion cell's spikes.

Figure 2. The visual feature of an amacrine pathway. (A) The linear filter and nonlineairty between a visual stimulus and a sustained Off amacrine cells membrane potential. This linear filter is refered to as the amacrine visual filter. (B) The transmission linear filter and nonlineairty between a current injected to an amacrine cell and a ganglion firing response. The amacrine pathway filter is obtained by cascading the amacrine visual filter and the amacrine transmission filter after compensating for the amacrine membrane voltage time constant. (C) From left to right: the first two principal components of 39 significant amacrine transmission filters (accounting for $64 \%$ of the variance); projection of all amacrine transmission filters onto these two principal components, colored similarly as in panels A-B; significant transmission filters color-coded according to their projection angle from the middle plot. (D) From left to right: the first two principal components of 39 amacrine pathway filters (accounting for $84 \%$ of the variance); projection of all amacrine pathway filters onto these two principal components (the points are coded by eight possible colors based on their angle around the origin of the two principal components plane); amacrine pathway filters color-coded according to their projection angle from the middle plot. (E) From left to right: the first two principal components of ganglion visual filters (accounting for $66 \%$ of the variance) based on responses of 104 recorded ganglion cells with firing rate greater than $0.5 \mathrm{~Hz}$; projection of all ganglion cell filters onto these two principal components (the points are coded by eight possible colors based on their angle around the origin of the two principal components plane); visual filters of the recoded ganglion cells color-coded according to their projection angle from the middle plot. (F) Illustrates a subset of points in the projection scatter diagram and their corresponding subset of ganglion visual filters 
Nonlinear effects of retinal amacrine cells

from (E) for the cases that a significant transmission filter was measured between an amacrine ganglion cell pair. The significance of a transmission is judged by a peak in the filter and/or by comparison with a shuffled data.

\section{Figure 3. Range of contributions of amacrine cells to the average ganglion cell visual}

feature. (A) Illustration of a model containing two main pathways, an amacrine pathway whose feature is shown by the red linear filter, and another pathway representing other ganglion cell features whose average is shown by the blue linear filter. The output of these two pathways are combined by a two-dimensional nonlinear function to generate the firing rate of ganglion cell. (B) (Top) Sample amacrine-ganglion cell pair for which amacrine pathway feature (red curve) does not contribute to the ganglion cell's STA (black curve) as indicated by the observation that the STA is the same as the oSTA (blue curve). (Bottom) The distribution of raw stimulus distribution (light gray) and the spike-triggered stimulus ensemble distribution (green), as well as the amacrine pathway nonlinear response function (computed as a quotient of the spike-triggered and raw stimulus distributions) (magenta), when the stimulus is projected onto the amacrine pathway feature (red). (C) Same as B for an amacrine cell that contributed to the STA of a ganglion cell. (D) Histogram of the observed similarities between the STA and oSTA for 39 amacrine-ganglion cell pairs, representing the range of the amacrine cells' linear contribution to the average temporal feature for the ganglion cell $(15.05 \pm 7.71$ degrees (mean $\pm S D))$. Greater distance between STA and oSTA indicates larger linear contribution from amacrine cell. Colored symbols indicate the angle differences for the cell pairs shown in B and C. (E) Difference between the time to peak of an amacrine pathway filter and the target ganglion cell for amacrine cells that contribute to the visual feature of the ganglion cell or not (contributing: $2.57 \pm 0.93 \mathrm{~ms}$ 
Nonlinear effects of retinal amacrine cells

(SEM), $\mathrm{n}=28$; non-contributing: $23.27 \pm 4.5 \mathrm{~ms}(\mathrm{SEM}) ; \mathrm{n}=11$; two-sample $\mathrm{t}$ test, $\mathrm{p}$-value < 0.001). (F) Temporal dynamics of ganglion visual filters (left), amacrine transmission filters (middle), and amacrine pathway filters (right), for 39 amacrine-ganglion cell pairs where the amacrine cell contributes to the visual feature of the ganglion cell or not (different colors). Principal components used here are the same as those in Figure 2.

Figure 4. A model using the measured amacrine pathway more accurately predicts the ganglion cell response. (A) Diagram of the two-pathway model used to predict the ganglion cell's firing rate response. The response of a ganglion cell was fit to the visual stimulus with a model containing two main pathways. The left pathway is the amacrine pathway visual feature (red filter), obtained by cascading the amacrine temporal receptive field and amacrine transmission kinetics. The right pathway is the average of other visual features encoded by the ganglion cell excluding the feature conveyed by the amacrine cell. Then, the outputs of these two pathways were combined through a two-dimensional instantaneous firing rate nonlinarity to predict the response of the ganglion cell. (B) Illustrates the prediction of the model in (A). The actual firing rate (black) is plotted together with the predicted firing rate of a ganglion cell for a 2-s stimulus segment from the test data (not used for fitting the model). (C) The correlation coefficient between the ganglion cell firing rate for two-pathway model in (A), a one-pathway LN model, and a two-pathway model using STA and the most significant STC feature orthogonalized to STA. The model using the amacrine pathway accurately predicts responses of ganglion cells $(n=39)$ to a novel white noise visual stimulus with a performance $(0.75 \pm 0.10)$ greater than that of the widely used LN model $(0.53 \pm 0.11)$ and on par with the performance of the two-pathway model using STC method $(0.78 \pm 0.07)$. 
Nonlinear effects of retinal amacrine cells

Figure 5. Differential modulation by an amacrine cell on multiple distinct ganglion cell visual features. (A) The multi-pathway model: The space of visual features encoded by a ganglion cell was decomposed into its principal components using STC analysis on the stimulus space when the amacrine pathway feature is projected out. Then for each of the ganglion cell's dimension and the amacrine pathway dimension a two-dimensional nonlinear firing rate function is computed. (B) (Left) Illustrates a nonlinear interaction between the amacrine pathway and one of the orthogonal STC features in (A) for a pair of an amacrine cell and a ganglion cell. Red plot represents the amacrine pathway feature; black plot represents the orthogonal STC feature; and green traces show one-diemnsional nonlinear response functions computed from the twodimensional instantaneous firing rate nonlinearity for three bins of amacrine pathway output values. (C) Eigenvalue profile of the STC matrix of the stimulus orthogonal to the amacrine cell pathway. For this sample amacrine-ganglion cell pair four significant STC dimensions corresponding to four significant eigenvalues were found. The total stimulus dimension was 60 stimulus frames over time in this example. (D) Amacrine-ganglion cell's features nonlinearity characterization: (Top) Orthogonal STC significant dimensions representing the ganglion cell's other features, excluding the amacrine pathway feature for a sample amacrine-ganglion cell pair in (C). (Middle) Two-dimensional firing rate nonlinearity as a function of the amacrine pathway output and the projection of the stimulus on each orthogonal feature. Lighter regions correspond to higher ganglion firing rate (FR) in the two-dimensional stimulus subspace. The $y$-axis is the projection of stimuli on the amacrine pathway dimension, and the $\mathrm{x}$-axis is the projection of the stimuli on the corresponding ganglion cell's recovered dimension. The two-dimentional instantaneuous firing function was computed as a quotient of the spike-conditional and raw 
Nonlinear effects of retinal amacrine cells

stimulus distributions. (Bottom) One-dimensional slices of each two-dimensional firing rate as a function of the projected stimulus onto the corresponding ganglion cell's feature for different levels of the amacrine cell's feature output. Trace color indicates different levels of the amacrine feature output. Because the amacrine cell is an Off cell, a high level of the preferred feature roughly corresponds to the amacrine cell being more hyperpolarized.

Figure 6. Diverse modulatory effects of amacrine cells on the ganglion cell population. (A) Illustrations of how changes in threshold, sensitivity, gain, offset and polarity reversal can be obtained by simple additive or multiplicative operations on ganglion cell's nonlinear response function. (B) (Left) Computational framework that can capture the modulatory effects of amacrine cell based on how the parameters in the ganglion cell's synaptic threshold function are tuned by the output of amacrine pathway. (Right) Potential mechanistic model for how computational effects might be implemented by presynaptic input, which would be delivered prior to the threshold of the bipolar cell synapse or postsynaptic input. Such an effect would cause a horizontal shifting or scaling of the sigmoidal response curve. A postsynaptic additive or multiplicative effect would cause a vertical shifting or scaling. (C) Six example different classes of nonlinear modulatory effects were observed across 150 amacrine-ganglion cell's feature pairs, which include combinations of additive and multiplicative transformations of the ganglion cell's nonlinear response function as the output of amacrine pathway varied. Additive inhibition could appear with other effects as well. (D) Example of modulatory effects of amacrine cells on ganglion cell visual feature. Each column is one cell pair, with different panels showing different visual features for the same ganglion cell. Panels above the dotted line represents a visual excitatory feature (positive eigenvalue), below the dotted line represents a suppressive visual 
Nonlinear effects of retinal amacrine cells

feature (negative eignevalue). The colored squares in each box denote the class of modulatory effects from (C), sensitivity, threshold etc., that was detected for each sample amacrine-ganglion feature pair.

\section{Figure 7. Amacrine modulatory effects across the ganglion cell population described by a}

multipathway model framework. (A) Fitting results using a quadratic-logistic nonlinear model for the ganglion cell's response function at different amacrine polarization levels. (B) Pairwise distribution of estimated parameters for 3 different amacrine polarizations, denoted by three shades of green color, showing how joint distribution of parameters change when the amacrine pathway output changes across 104 amacrine-ganglion feature pairs for which the logistic nonlinear function could fit the data nonlinearity. Histograms along each plot axis represent the distribution of the parameter values along that axis for three different amacrine polarizations $(\alpha$ : $[5.74 \pm 0.725 .08 \pm 0.704 .6 \pm 0.62], \beta:[2.04 \pm 1.611 .84 \pm 1.411 .95 \pm 1.49], \gamma:[-0.05 \pm 0.45-$ $0.05 \pm 0.36-0.05 \pm 0.33]($ mean $\pm \mathrm{SD}))$. Each grey line indicates the direction of the change along the $\mathrm{x}$ - and $\mathrm{y}$-axes from the most depolarized amacrine pathway output to the most hyperpolarized amacrine pathway output, and a longer line indicates a greater change and their directionality indcate the joint behavior of the two parameters. All grey lines are centered at the mean value of the $\mathrm{x}$-axis and $\mathrm{y}$-axis, indicating that amacrine depolarization for most cell pairs caused a change in $\alpha$ in one direction, but changes for $\beta$ and $\gamma$ could occur in either direction, and were uncorrelated with the effect on $\alpha$. (C) Scatter plots of pairwise normalized changes in estimated parameters show diversity in how the amacrine pathway can affect the parameters of the model. Each data point $(n=104)$ represents the slope of a least-squares line fit to the values of each parameter estimated across different levels of amacrine pathway polarization, normalized 
Nonlinear effects of retinal amacrine cells

by standard deviation of the distribution of each parameter's values. The amacrine pathway output is quantized into three levels from highly depolarized to highly hyperpolarized for these plots. Histograms along each plot axis represent the distribution of the normalized values along that axis (normalized $\Delta \alpha=-2.24 \pm 1.0$, normalized $\Delta \beta=-0.05 \pm 0.59$, normalized $\Delta \gamma=0 \pm 0.11$ (mean \pm SD)). The light gray ellipses around each point indicate the uncertainty of the parameters as evaluated by a five-fold cross-validation procedure. The distrbution of the variability in parameter estimation indicate that the model is robust given the data. Schematic nonlinearities illustrate the range of nonlinearity shapes that the model can fit. For some amacrine-ganglion feature pairs the nonlinearity was not monotonic and a combination of scaling/shifting in both horizontal and vertical direction were observed. (D) Histogram of the sensitivity of the three nonlinearity parameters to the different levels of amacrine polarizations. The parameter sensitivity was measured as the percentage of times in which the slope of the least-squares line fit to values of a parameter was significant.

Figure S1 (related to figure 2). Diversity of amacrine transmission dynamics for single amacrine cells versus multiple ganglion cells. Each plot shows the significant transmission filters from one amacrine cell to the affected ganglion cells in an experiment, for four sample amacrine cells. The colors represent the type of transmission filter as classified according to the principal component analysis in figure $2 \mathrm{C}$.

Figure S2 (related to figure 2 and figure 6). Diversity both in linear transmissions and in nonlinear modulatory effects from one amacrine cell to multiple ganglion cells. The plots illustrate the linear and nonlinear interactions between one sample amacrine cell versus five 
Nonlinear effects of retinal amacrine cells

ganglion cells. Each row represent a pair of amacrine and ganglion cells where the amacrine cell is the same across rows. Red waveforms (first column) represent amacrine pathway filters between the amacrine cell and each ganglion cell. Black waveforms (second column) represent ganglion visual filters for each five affected ganglion cells in the experiment. Green traces (third column) show ganglion firing rate as a function of projection of stimulus on the ganglion visual features with the amacrine pathway feature excluded.

Figure S3 (related to figure 5). Significance test of STC features. (A) Eigenvalue spectrum is computed for different fractions of data, ranging from one-tenth to the entire number of spikes. Those eigenvalues that remain stable across different fraction of data (sample green trace) are considered as significant eigenvalues, i.e., those that are different from the bulk spectrum due to the spiking events not due to random fluctuations along poorly sampled dimensions. Those eigenvalues that vanish (sample brown traces) when the size of data increases are considered as nonsignificant. (B) Nested bootstrap method was performed to test the significance of each eigenvalue. The null hypothesis starts with the assumption that there is no significant eigenvalue, then a confidence interval for each eigenvalue is computed, if at least one eigenvalue falls outside of the confidence interval the null hypothesis is rejected (left plot), and the next null hypothesis with the assumption of only one significant eigenvalue is tested, and this procedure continues until a null hypothesis is accepted (right plot), i.e. assuming the null hypothesis, no eigenvalue falls outside of the confidence interval. For the example shown here four significant eigenvalues were found. 
Nonlinear effects of retinal amacrine cells

\section{EXPERIMENTAL PROCEDURES}

\section{Electrophysiology}

\section{Visual Stimulation}

Visual stimuli were projected onto the retina from a video monitor, at a photopic mean intensity of $8 \mathrm{~mW} / \mathrm{m}^{2}$, and at a frame rate of $63 \mathrm{~Hz}$. The video monitor was calibrated using a photodiode to ensure the linearity of the display. Stimuli were spatially uniform and were drawn from a White Noise Gaussian distribution with a constant mean intensity. Contrast was defined as the standard deviation divided by the mean of the intensity values. Contrast was fixed throughout the experiment, and ranged $0.06-0.25$ across recording sessions. The duration of stimuli was 300-1200 s. By keeping the mean light intensity and contrast constant throughout the experiment, we avoided any contributions from light or contrast adaptation (Baccus and Meister, 2002; Manu and Baccus, 2011).

\section{Simultaneous intracellular and multielectrode recording}

\section{Multielectrode Recording}

To record the spike trains of retinal ganglion cells, the retina of the larval tiger salamander was isolated intact and was placed on a dialysis membrane attached to a plastic holder. Then the holder was lowered onto a flat array of 61 microelectrodes (Multichannel Systems) and bathed in oxygenated Ringer's solution buffered with bicarbonate (Meister et al., 1994; Smirnakis et al., 1997). Extracellular electrodes were spaced $100 \mu \mathrm{m}$ apart. The electrical signals from the array of electrodes were digitized at $10 \mathrm{kHz}$ and recorded to a computer. Results included 153 
Nonlinear effects of retinal amacrine cells

recorded ganglion cells, which comprised biphasic Off-type, monophasic Off-type, biphasic ontype, and On-Off cells classified with a uniform field visual stimulus.

\section{Intracellular Recording}

Simultaneous intracellular recordings were made with sharp electrodes from eleven amacrince cells with a resting membrane potential $-55 \pm 4 \mathrm{mV}$, mean $\pm \mathrm{SEM}$ ), including sustained $(n=8)$ and transient $(n=3)$ off amacrine cells. Amacrine cells were identified by their light responses, the presence of an inhibitory surround, and their inhibitory transmission to Offtype ganglion cells with overlapping receptive field centers. The retina was held in place over the electrode array under a $100-\mu \mathrm{m}$ layer of $0.6 \%$ agarose, covered by a dialysis membrane containing several $100-\mu \mathrm{m}$ holes. The intracellular electrode was then guided under infrared light through a hole and the agarose layer to penetrate the retina from the photoreceptor side. Intracellular electrodes (150-250 M $\Omega$ impedance) were filled with 1-2 M potassium acetate.

\section{Current Stimulation}

Current was injected through sharp microelectrodes (150-250 M $\Omega$ ) with an amplifier operating in bridge mode, into individual amacrine cells. Current and the visual stimulus were aligned in time within $0.1 \mathrm{~ms}$, to allow reproducible recordings across the same visual and current stimuli presentations. The current amplitudes were chosen so they maintain the membrane potential within a physiological range. The estimated membrane conductance measured by using pulses of current was $40 \pm 17 \mathrm{M} \Omega$, and the measured membrane time constant was $17 \pm 7 \mathrm{~ms}(\mathrm{n}=6)$. The current with $0-50 \mathrm{~Hz}$ bandwidth was convolved with an exponential filter of the appropriate amplitude to estimate the resulting membrane potential standard deviation (9.6 $\pm 3.63 \mathrm{mV})$ (Manu and Baccus, 2011). 
Nonlinear effects of retinal amacrine cells

\section{Analysis}

The significance level is 0.05 in all analyses.

\section{Measuring linear-nonlinear cascade models of the recorded responses}

Recordings were used to produce LN models of visual responses and of responses to current injection (Baccus and Meister, 2002). The input to the model could be a visual stimulus or a current stimulus, and the output of the model could be either the predicted ganglion cell's firing rate or the predicted amacrine cell's membrane voltage. The estimated linear filters ranged from 200 to $600 \mathrm{~ms}$ in duration, and were estimated using white noise stimuli of length $300-1200 \mathrm{~s}$.

To estimate the parameters of the LN model we used a semiparametric approach, in which the filter $f(t)$ and the nonlinearity $N(g)$ were computed at once. For a Gaussian white noise input, the optimal linear filter is indeed the Wiener system solution, which was computed in the Fourier domain by equation (1) (Bussgang, 1952; Hunter and Korenberg, 1986).

$\tilde{F}(\omega)=\frac{\left\langle\tilde{s}^{*}(\omega) \tilde{r}(\omega)\right\rangle}{\left\langle\tilde{s}^{*}(\omega) \tilde{s}(\omega)\right\rangle}$

where $\tilde{s}(\omega)$ is the Fourier transform of the stimulus $s(t), \tilde{s}^{*}(\omega)$ its complex conjugate, $\tilde{r}(\omega)$ is the Fourier transform of the response $r(t)$, and $\langle\ldots\rangle$ denotes averaging over $1 \mathrm{~s}$ segments spaced every $0.1 \mathrm{~s}$ throughout the recording (Baccus and Meister, 2002). The stimulus intensity $s(t)$ was adjusted to have a zero mean. The denominator is the input auto-correlation, which corrects for possible deviation from the white Gaussian solution due to possible correlation structures in the input. For fitting of the ganglion cell spiking response, spike trains were converted into a continuous firing rate by binning the spike times in $1 \mathrm{~ms}$ intervals. 
Nonlinear effects of retinal amacrine cells

The predicted linear ganglion cell's firing rate response or the amacrine cell's membrane voltage $g(t)$ was computed by convolving $f(t)$ with the stimulus $s(t)$.

$g(t)=\int F(\tau) s(t-\tau) d \tau$

Then, a nonlinear function $N(g)$ mapping the response to the linear prediction $g(t)$ was found by computing the average value of $r(t)$ over bins of $g(t)$ containing an equal number of points. The output of $N(g)$ determines the instantaneous firing rate of a Poisson spike generator (Chichilnisky, 2001).

The amplitude of the filter was normalized such that the variance of the filtered stimulus, $g(t)$, was equal to the variance of the stimulus, $s(t)$ (equation 3), to avoid the ambiguity existing in the scaling of linear filter $y$-axis and the nonlinearity $x$-axis.

$\int g^{2}(\tau) d \tau=\int s^{2}(\tau) d \tau$

With this normalization, $f(t)$ summarizes temporal processing, and $N(g)$ captures the sensitivity of the stimulus (Baccus and Meister, 2002).

Finally, the prediction of the LN model was calculated as

$r^{\prime}(t)=N(g(t))=N\left(\int F(\tau) s(t-\tau) d \tau\right)$

Spikes are then assumed to be generated by a Poisson process with a rate equal to $N(g)$.

\section{Computation of amacrine pathway feature}

Amacrine visual response LN models were constructed using a white noise visual stimulus with a Gaussian distribution and the amacrine's membrane potential recorded intracellularly (Fig. 2A). Similarly, a linear filter corresponding to amacrine cell transmission was computed by correlating the white noise Gaussian current signal with the spiking response of the ganglion cell, 
Nonlinear effects of retinal amacrine cells

and then a static nonlinearity was computed by comparing the current linear prediction and the firing rate of the ganglion cell. These linear and nonlinear stages together were called the amacrine cell's transmission LN model (Fig. 2B). Since the injected current and the visual stimulus were uncorrelated, the visual stimulus could be excluded in computing the amacrine transmission filter.

The output of the first LN model was amacrine's membrane voltage produced by the current generated by presenting the visual stimulus to the amacrine cell. However, the input to the second LN model was the white noise Gaussian current injected to the amacrine cell, which by itself induced a voltage across the amacrine's membrane. So in order to compensate for the discrepancy in the output of amacrine visual response LN model and the input of the measured transmission filter, we deconvolved the measured transmission filter by the amacrine's membrane time constant (equation 5), to get the amacrine voltage transmission filter (Manu and Baccus, 2011); the membrane time constant of the cell was measured by applying current pulses of $1 \mathrm{~s}$. An exponential filter (equation 5) was fit to the voltage response with membrane time constant $\tau$.

$F_{m}(t)=e^{-t / \tau}$

\section{Computing the output of amacrine pathway}

Once the complete amacrine pathway model was obtained, the output signal of the amacrine pathway was computed during the current injection, which was used to study the effect of amacrine pathway on the response of the ganglion cells. During current injection, the amacrine membrane voltage was produced by two sources of currents including the white noise Gaussian current injected to the amacrine cell and the current generated by the visual stimulation of the 
Nonlinear effects of retinal amacrine cells

amacrine cell. We assumed for these two currents to be linearly combined in the amacrine cell before transmission to the ganglion cell, where the magnitude of their individual contributions (linear weights) was obtained by an additive least-squares model. This summed current was then convolved with the voltage transmission filter and then passed through the transmission nonlinearity.

\section{STA and STC analysis}

The STA represents the difference between the mean (center of mass) of the spike-triggered ensemble and the mean of the raw stimulus ensemble and is estimated as follows (assuming that the raw stimuli have zero mean):

$\hat{A}=\frac{1}{N} \sum_{n=1}^{N} \vec{s}\left(t_{n}\right)$

where $t_{n}$ is the time of the $n^{\text {th }}$ spike, $\vec{s}\left(t_{n}\right)$ is a vector representing the stimuli preceding $t_{n}$, and $N$ is the total number of spikes (Schwartz et al., 2006).

For a neuron with a single linear filter, the STA provides an unbiased estimate of the neuron's linear filter (as obtained by reverse correlation in equation 1), provided that the input has a spherically symmetric distribution, like a white Gaussian stimuli (Bussgang, 1952; Chichilnisky, 2001; Paninski, 2003; Schnitzer and Meister, 2003; Simoncelli et al., 2004; Theunissen et al., 2001), and the nonlinearity of the model is such that it leads to a shift in the mean of the spiketriggered ensemble relative to the raw stimulus ensemble.

To capture multiple features, the spike-triggered covariance (STC) technique was used (Brenner et al., 2000a; Rust et al., 2005; Simoncelli et al., 2004), where additional filters were recovered by seeking directions in the stimulus space in which the variance of the spike-triggered 
Nonlinear effects of retinal amacrine cells

ensemble differs from that of the raw ensemble. Unlike the change in the mean that defines a single dimension, the variance can recover many dimensions at once.

The covariance matrix is formed from the outer product of the stimulus histories averaged across all spikes as follows.

$\hat{C}_{\text {post }}=\frac{1}{N-1} \sum_{n=1}^{N}\left(\vec{S}\left(t_{n}\right)-\hat{A}\right)\left(\vec{S}\left(t_{n}\right)-\hat{A}\right)^{T}$,

where $t_{n}$ is the time of the $n^{\text {th }}$ spike, $\vec{s}\left(t_{n}\right)$ is a vector representing the stimuli preceding $t_{n}$, and $N$ is the total number of spikes, and $\hat{A}$ is the STA (as computed in equation 6) (Schwartz et al., 2006).

$\hat{C}_{\text {post }}$ contains the multidimensional variance structure of the spike-triggered stimulus ensemble, such that the variance of the STE along any direction $u$ ( $u$ is a unit vector) is $u^{T} \hat{C}_{\text {post }} u$. We were interested in finding the features in the stimulus space for which the variance changes from that of the prior distribution of stimuli, which is Gaussian along all stimulus dimensions, independent of the response. We first construct the covariance difference matrix by subtracting $\hat{C}_{\text {prior }}$ from $\hat{C}_{\text {post }}$,

$\Delta C=\hat{C}_{\text {post }}-\hat{C}_{\text {prior }}$

where $\hat{C}_{\text {prior }}=\sum_{n} \vec{S}\left(t_{n}\right) \vec{s}\left(t_{n}\right)^{T}$.

Then the posterior second moment matrix $\hat{C}_{\text {post }}$ can in general only differ from $\hat{C}_{\text {prior }}$ in directions spanned by the model filters (Brenner et al., 2000b). An eigenvector analysis of this matrix can determine the stimulus directions that account for most of the variance.

\section{Computation of orthogonal features}


Nonlinear effects of retinal amacrine cells

To model the other parts of the circuit feeding into the ganglion cell, we focused on the subset of stimuli that were not encoded by the amacrine cell pathway. To do this, we orthogonalized the stimulus space with respect to the direction of the total amacrine pathway feature using a Gram-Schmidt procedure. Then we computed the ganglion cell's STA in this new stimulus space as follows,

$\widehat{A^{\prime}}=\frac{1}{N} \sum_{n=1}^{N} \overrightarrow{s^{\prime}}\left(t_{n}\right)$

where $\overrightarrow{s^{\prime}}=\vec{s}-\langle\vec{s}, \vec{f}\rangle \vec{f} /\|\vec{f}\|,\langle\ldots\rangle$ denotes the inner products of two vector arguments, and $\vec{f}$ represents the amacrine pathway feature. We refer to $\widehat{A}^{\prime}$ as ganglion cell's orthogonal STA (oSTA).

Similarly, we used the STC approach to recover the entire linear subspace representing the space spanned by the features encoded by the multiple pathways feeding into a ganglion cell that does not include the amacrine pathway feature. To find such features, we first projected out the amacrine pathway feature from the stimulus space, and construct the sample second moment matrix of the spike-triggered ensemble in the new stimulus space and the covariance difference matrix and found its eigenvectors and corresponding eigenvalues as explained above.

These eigenvectors identify the dimensions in the stimulus space along which the variance of spike-triggered stimulus distribution changes from that of the prior distribution of stimuli. Therefore, each eigenvector of this matrix represents a visual feature that might elicit spikes in the ganglion cell. The associated eigenvalue is equal to the variance of spike-triggered stimuli along this feature direction. Because the prior stimulus covariance matrix is subtracted from the posterior stimulus covariance matrix, an eigenvalue of zero corresponds to the prior variance and indicates that its associated eigenvector is not a relevant feature for ganglion cell response. So to 
Nonlinear effects of retinal amacrine cells

find the relevant subspace, we extract the eigenvalues of the difference covariance matrix that are significantly different from zero. The number of significant eigenvalues gives an estimate for the dimensionality of the relevant stimulus space.

\section{Assessing the number of significant orthogonal ganglion cell features}

In the absence of any relationship between the stimulus and the spikes, the variance of the spike-triggered ensemble would be identical to that of the raw stimulus ensemble, i.e., when we project the stimulus along an irrelevant dimension, in the limit of infinite sampling, the resulting spike triggered stimulus ensemble would be only a random subset of the raw stimulus ensemble. However, in a real experiment, due to the finite sampling we saw random fluctuations in the variances in different directions. But we were interested in recovering only relevant features, i.e., those dimensions in the stimulus space along which we saw an increase or decrease in the variance of the spike-triggered ensemble that was greater than the variance change due to finite sampling. Figure 5C shows the eigenvalue profile for a sample amacrine-ganglion cells pair, in which the eigenvalues of the covariance difference matrix were sorted from large to small values. The majority of the eigenvalues descend gradually indicating random fluctuations due to finite sampling issue (the white noise covariance difference matrix should have had constant eigenvalues at zero).

To assess the significance of eigenvalues we adopted a variety of the significance tests, as sometimes the insufficient number of samples along some dimensions due to the low firing rate of the neuron, caused noisy estimations for the confidence intervals. In general, we preferred more qualitative definitions of significance, i.e., choose all eigenvectors corresponding to eigenvalues, which appear qualitatively different in magnitude from the "bulk spectrum" 
Nonlinear effects of retinal amacrine cells

(Johnstone, 2000) (Fig. 5C). Even this simple approach could provide a consistent estimator for the relevant subspace in the case of a standard Gaussian input stimulus (Paninski, 2003). However, due to orthogonalization there were cases that we could detect eigenvalues, which were significantly below or above the gradually descending region, but with arbitrary associated eigenvectors. For these cases, we used quantitative significance tests and concluded about the significance where the distinction was not so clear. We assessed eigenvalue significance by computing the eigenvalue spectrum as a function of the number of spikes for a range of data fractions (y Arcas et al., 2003). Eigenvalues that were stable with respect to the number of spikes used in the analysis were judged to be significant (green trace in Fig. S3A). We also used a nested hypotheses testing approach to determine the number of relevant dimensions corresponding to significant increases or decreases in variance (Rust et al., 2005). This nested approach was necessary as the distribution of the lowest and highest eigenvalues under the null hypothesis depended on the dimensionality of the space. Figure S3B shows the nested bootstrapping procedure for a sample amacrine-ganglion cells pair. Thus, both the quality and number of filters recovered by STC depend not only on the strength of their influence on neural response, but also on the number of spikes collected (Paninski, 2003; y Arcas and Fairhall, 2003). So the number of significant eigenvalues we obtained by these methods is a lower bound to the true dimensionality of the subspace of relevant ganglion cell's features. To get more accurate results, we only included ganglion cells from which we recorded at least 1200 spikes that is at least 20 spikes per temporal dimension for 60-dimensional linear temporal filters. We used 60 stimulus values preceding a spike. Thus for stimuli presented at a frame rate of $63 \mathrm{~Hz}$, the stimulus $s(t)$ extended back to $960 \mathrm{~ms}$ before a spike.

\section{Parameterization of amacrine nonlinear effects}


Nonlinear effects of retinal amacrine cells

Our goal was to find a model of modulatory functions of amacrine cells, which is consistent with a set of experimentally estimated correlations between the stimulus and neuron's response, and at the same time imposes as little structure as possible for the nonlinear interactions among different pathways.

To this end, we estimated the ganglion nonlinear response function using the maximum entropy framework borrowed from statistical mechanics (Jaynes, 1957a, b). According to the maximum entropy principle, to obtain the least-biased model of a given set of data, we must choose the distribution that has the maximal entropy, i.e. is minimally constrained, among all the distributions that may be consistent with the data. It has been suggested that such a model has the best performance in predicting a novel or unobserved set of data (Jaynes, 2003). This approach has been recently used to characterize the responses of neural populations (Schneidman et al., 2006; Shlens et al., 2006). On the other hand, for a system with a binary output (such as spike trains) and a given set of constraints on the input-output moments, the maximum entropy models are logistic functions whose arguments depend on the correlation constraints. A constraint on the average output turns these models into minimum mutual information models, which allows for an information theoretic characterization of the system's computations (Fitzgerald et al., 2011).

Thus, inspired by the maximum entropy methods, we used a set of quadratic logistic functions (equation 10) to describe the nonlinear interactions between amacrine pathway dimension and other ganglion cell dimensions.

$$
P\left(r_{i}=1 \mid g_{i}\right)=\frac{1}{1+\exp \left[\alpha+\beta\left(g_{i}-\gamma\right)^{2}\right]}
$$

where $g_{i}$ is the projection of stimulus on one of the ganglion cell's orthogonal feature dimension at time $i$, and $\alpha, \beta, \gamma$ are parameters to be estimated. The response $r_{i}$ is obtained by binning the 
Nonlinear effects of retinal amacrine cells

ganglion spike trains such that $r_{i}=1$ if there was at least one spike in bin $i$, and $r_{i}=0$ otherwise.

We fit this function to the ganglion cell's response at different levels of amacrine polarization and used the estimated parameters to quantify the modulatory effects of the amacrine pathway.

Assuming a Poisson like spiking response, the probability of observing a sequence of spikes $r_{1}, r_{2}, \cdots, r_{i}, \cdots$ is the product over $i$ of $p_{i}^{r_{i}} \cdot\left(1-p_{i}\right)^{1-r_{i}}$, where $p_{i}$ is the probability of spiking at time $i$. Then, the log-likelihood is:

$$
L(\alpha, \beta, \gamma)=-\sum_{i} r_{i}\left[\alpha+\beta\left(g_{i}-\gamma\right)^{2}\right]-\sum_{i} \ln \left[1+\exp \left(-\alpha-\beta\left(g_{i}-\gamma\right)^{2}\right)\right]
$$

The optimal values of $\alpha, \beta, \gamma$ are then obtained by minimizing $-L$, which is the negative of log-likelihood function. None of the parameters in this optimization problem have a constrained range. Finding appropriate initial parameter values therefore involves experimentation. We often used $\gamma_{0}=$ mean $(g) ; \beta_{0}= \pm 1 / \operatorname{var}(g) ; \alpha_{0}=\ln \left[\frac{1-P\left(r=1 \mid g=\gamma_{0}\right)}{P\left(r=1 \mid g=\gamma_{0}\right)}\right]$. The appropriate sign of $\beta_{0}$ depends on whether the nonlinearity looks Gaussian-like $(\beta>0)$ or U-shaped $(\beta<0)$.

\section{Cross-validation of the nonlinear model parameters}

To distinguish between the variability in the change of parameters across amacrine ganglion cell feature pairs and the variations from noise, we performed a cross-validation procedure for the parameter estimation: data was divided into five randomly assigned subsets; at each iteration, $80 \%$ of data was used to estimate the parameters and the remaining $20 \%$ of data was used to evaluate the goodness of fit in terms of the log-likelihood of the observed ganglion response; the resulting five sets of parameters were used to find the standard error in estimating each parameter. The results in figure $7 \mathrm{C}$ indicate that the error bars associated with each 
bioRxiv preprint doi: https://doi.org/10.1101/273730; this version posted February 28, 2018. The copyright holder for this preprint (which was not certified by peer review) is the author/funder. All rights reserved. No reuse allowed without permission.

Nonlinear effects of retinal amacrine cells

estimated parameter are smaller than variations across data points, confirming that many different behaviors are observed that form a continuum of distinct effects. 
Nonlinear effects of retinal amacrine cells

\section{REFERENCES}

Asari, H., and Meister, M. (2012). Divergence of visual channels in the inner retina. Nature neuroscience $15,1581-1589$.

Asari, H., and Meister, M. (2014). The projective field of retinal bipolar cells and its modulation by visual context. Neuron 81, 641-652.

Atencio, C.A., Sharpee, T.O., and Schreiner, C.E. (2008). Cooperative nonlinearities in auditory cortical neurons. Neuron 58, 956-966.

Atick, J.J., and Redlich, A.N. (1990). Towards a theory of early visual processing. Neural Computation 2, 308-320.

Atick, J.J., and Redlich, A.N. (1993). Convergent algorithm for sensory receptive field development. Neural Computation 5, 45-60.

Attneave, F. (1954). Some informational aspects of visual perception. Psychological review 61, 183.

Baccus, S.A. (2007). Timing and computation in inner retinal circuitry. Annu Rev Physiol 69, 271-290.

Baccus, S.A., and Meister, M. (2002). Fast and slow contrast adaptation in retinal circuitry. Neuron 36, 909-919.

Baccus, S.A., Ölveczky, B.P., Manu, M., and Meister, M. (2008). A retinal circuit that computes object motion. The Journal of Neuroscience 28, 6807-6817.

Barlow, H. (2001). Redundancy reduction revisited. Network: computation in neural systems 12, 241-253.

Barlow, H.B. (1961). Possible principles underlying the transformations of sensory messages. 
Nonlinear effects of retinal amacrine cells

Bell, A.J., and Sejnowski, T.J. (1997). The "independent components" of natural scenes are edge filters. Vision research 37, 3327-3338.

Berry, M.J., Warland, D.K., and Meister, M. (1997). The structure and precision of retinal spike trains. Proceedings of the National Academy of Sciences 94, 5411-5416.

Bialek, W., and van Steveninck, R.R. (2005). Features and dimensions: Motion estimation in fly vision. arXiv preprint q-bio/0505003.

Brenner, N., Bialek, W., and Van Steveninck, R.d.R. (2000a). Adaptive rescaling maximizes information transmission. Neuron 26, 695-702.

Brenner, N., Strong, S.P., Koberle, R., Bialek, W., and Van Steveninck, R.R.D.R. (2000b). Synergy in a neural code. Neural computation 12, 1531-1552.

Bussgang, J. (1952). Crosscorrelation functions of amplitude-distorted Gaussian signals. Research Lab. Electron. (MIT, Cambridge, MA, USA, Tech. Rep).

Cantrell, D.R., Cang, J., Troy, J.B., and Liu, X. (2010). Non-centered spike-triggered covariance analysis reveals neurotrophin-3 as a developmental regulator of receptive field properties of ONOFF retinal ganglion cells. PLoS Comput Biol 6, e1000967.

Carandini, M., and Heeger, D.J. (2012). Normalization as a canonical neural computation. Nature Reviews Neuroscience 13, 51-62.

Carandini, M., Heeger, D.J., and Movshon, J.A. (1997). Linearity and normalization in simple cells of the macaque primary visual cortex. The Journal of Neuroscience 17, 8621-8644.

Carvalho, T.P., and Buonomano, D.V. (2009). Differential effects of excitatory and inhibitory plasticity on synaptically driven neuronal input-output functions. Neuron 61, 774-785.

Chance, F.S., Abbott, L., and Reyes, A.D. (2002). Gain modulation from background synaptic input. Neuron 35, 773-782. 
Nonlinear effects of retinal amacrine cells

Chen, X., Han, F., Poo, M.-m., and Dan, Y. (2007). Excitatory and suppressive receptive field subunits in awake monkey primary visual cortex (V1). Proceedings of the National Academy of Sciences 104, 19120-19125.

Chen, X., Hsueh, H.-A., Greenberg, K., and Werblin, F.S. (2010). Three forms of spatial temporal feedforward inhibition are common to different ganglion cell types in rabbit retina. Journal of neurophysiology 103, 2618-2632.

Chichilnisky, E. (2001). A simple white noise analysis of neuronal light responses. Network: Computation in Neural Systems 12, 199-213.

Coen-Cagli, R., Dayan, P., and Schwartz, O. (2009). Statistical models of linear and nonlinear contextual interactions in early visual processing. In Advances in neural information processing systems, pp. 369-377.

de Vries, S.E., Baccus, S.A., and Meister, M. (2011). The projective field of a retinal amacrine cell. The Journal of Neuroscience 31, 8595-8604.

DeAngelis, G., Robson, J., Ohzawa, I., and Freeman, R. (1992). Organization of suppression in receptive fields of neurons in cat visual cortex. Journal of Neurophysiology 68, 144-163.

Dong, C.-J., and Hare, W.A. (2003). Temporal modulation of scotopic visual signals by A17 amacrine cells in mammalian retina in vivo. Journal of neurophysiology 89, 2159-2166.

Dong, D.W., and Atick, J.J. (1995). Statistics of natural time-varying images. Network: Computation in Neural Systems 6, 345-358.

Fairhall, A.L., Burlingame, C.A., Narasimhan, R., Harris, R.A., Puchalla, J.L., and Berry, M.J. (2006). Selectivity for multiple stimulus features in retinal ganglion cells. Journal of neurophysiology 96, 2724-2738.

Felsen, G., Touryan, J., Han, F., and Dan, Y. (2005). Cortical sensitivity to visual features in natural scenes. PLoS Biol 3, e342. 
Nonlinear effects of retinal amacrine cells

Field, D.J. (1987). Relations between the statistics of natural images and the response properties of cortical cells. JOSA A 4, 2379-2394.

Fitzgerald, J.D., Sincich, L.C., and Sharpee, T.O. (2011). Minimal models of multidimensional computations. PLoS Comput Biol 7, e1001111.

Fox, J.L., Fairhall, A.L., and Daniel, T.L. (2010). Encoding properties of haltere neurons enable motion feature detection in a biological gyroscope. Proceedings of the National Academy of Sciences 107, 3840-3845.

Geffen, M.N., De Vries, S.E., and Meister, M. (2007). Retinal ganglion cells can rapidly change polarity from Off to On. PLoS Biol 5, e65.

Hallum, L.E., and Movshon, J.A. (2014). Surround suppression supports second-order feature encoding by macaque V1 and V2 neurons. Vision research 104, 24-35.

Heeger, D.J. (1992). Normalization of cell responses in cat striate cortex. Visual neuroscience 9, 181-197.

Hong, S., y Arcas, B.A., and Fairhall, A.L. (2007). Single neuron computation: from dynamical system to feature detector. Neural computation 19, 3133-3172.

Horwitz, G.D., Chichilnisky, E., and Albright, T.D. (2005). Blue-yellow signals are enhanced by spatiotemporal luminance contrast in macaque V1. Journal of Neurophysiology 93, 2263-2278.

Horwitz, G.D., Chichilnisky, E., and Albright, T.D. (2007). Cone inputs to simple and complex cells in V1 of awake macaque. Journal of Neurophysiology 97, 3070-3081.

Hubel, D.H., and Wiesel, T.N. (1962). Receptive fields, binocular interaction and functional architecture in the cat's visual cortex. The Journal of physiology 160, 106-154.

Hunter, I., and Korenberg, M. (1986). The identification of nonlinear biological systems: Wiener and Hammerstein cascade models. Biological cybernetics 55, 135-144. 
Nonlinear effects of retinal amacrine cells

Isaacson, J.S., and Scanziani, M. (2011). How inhibition shapes cortical activity. Neuron 72, 231-243.

Jadzinsky, P.D., and Baccus, S.A. (2013). Transformation of visual signals by inhibitory interneurons in retinal circuits. Annual review of neuroscience 36, 403-428.

Javel, E., Geisler, C.D., and Ravindran, A. (1978). Two-tone suppression in auditory nerve of the cat: Rate-intensity and temporal analyses. The Journal of the Acoustical Society of America 63, 1093-1104.

Jaynes, E.T. (1957a). Information theory and statistical mechanics. Physical review 106, 620.

Jaynes, E.T. (1957b). Information theory and statistical mechanics. II. Physical review 108, 171.

Jaynes, E.T. (2003). Probability theory: The logic of science (Cambridge university press).

Johnstone, I. (2000). On the distribution of the largest principal component (Department of Statistics, Stanford University).

Kapadia, M.K., Westheimer, G., and Gilbert, C.D. (1999). Dynamics of spatial summation in primary visual cortex of alert monkeys. Proceedings of the National Academy of Sciences 96, 12073-12078.

Karklin, Y., and Lewicki, M.S. (2005). A hierarchical Bayesian model for learning nonlinear statistical regularities in nonstationary natural signals. Neural computation 17, 397-423.

Katzner, S., Busse, L., and Carandini, M. (2011). GABAA inhibition controls response gain in visual cortex. The Journal of neuroscience 31, 5931-5941.

Kim, I.S., Choi, H.S., Yi, K.M., Choi, J.Y., and Kong, S.G. (2010). Intelligent visual surveillance - a survey. International Journal of Control, Automation and Systems 8, 926-939.

Kim, K.J., and Rieke, F. (2001). Temporal contrast adaptation in the input and output signals of salamander retinal ganglion cells. The Journal of Neuroscience 21, 287-299. 
Nonlinear effects of retinal amacrine cells

Knierim, J.J., and Van Essen, D.C. (1992). Neuronal responses to static texture patterns in area V1 of the alert macaque monkey. Journal of Neurophysiology 67, 961-980.

Levitt, J.B., and Lund, J.S. (1997). Contrast dependence of contextual effects in primate visual cortex. Nature 387, 73-76.

Machens, C.K., Wehr, M.S., and Zador, A.M. (2004). Linearity of cortical receptive fields measured with natural sounds. The Journal of neuroscience 24, 1089-1100.

Manu, M., and Baccus, S.A. (2011). Disinhibitory gating of retinal output by transmission from an amacrine cell. Proceedings of the National Academy of Sciences 108, 18447-18452.

Maravall, M., Petersen, R.S., Fairhall, A.L., Arabzadeh, E., and Diamond, M.E. (2007). Shifts in coding properties and maintenance of information transmission during adaptation in barrel cortex. PLoS Biol 5, e19.

Markram, H., Toledo-Rodriguez, M., Wang, Y., Gupta, A., Silberberg, G., and Wu, C. (2004). Interneurons of the neocortical inhibitory system. Nature Reviews Neuroscience 5, 793-807.

Masland, R., Mills, J., and Cassidy, C. (1984). The functions of acetylcholine in the rabbit retina. Proceedings of the Royal Society of London B: Biological Sciences 223, 121-139.

Masland, R.H. (2001). The fundamental plan of the retina. Nature neuroscience 4, 877-886.

Masland, R.H. (2012a). The neuronal organization of the retina. Neuron 76, 266-280.

Masland, R.H. (2012b). The tasks of amacrine cells. Visual neuroscience 29, 3-9.

Meister, M., Pine, J., and Baylor, D.A. (1994). Multi-neuronal signals from the retina: acquisition and analysis. Journal of neuroscience methods 51, 95-106.

Mitchell, S.J., and Silver, R.A. (2003). Shunting inhibition modulates neuronal gain during synaptic excitation. Neuron 38, 433-445. 
Nonlinear effects of retinal amacrine cells

Molnar, A., Hsueh, H.-A., Roska, B., and Werblin, F.S. (2009). Crossover inhibition in the retina: circuitry that compensates for nonlinear rectifying synaptic transmission. Journal of computational neuroscience 27, 569-590.

Molnar, A., and Werblin, F. (2007). Inhibitory feedback shapes bipolar cell responses in the rabbit retina. Journal of neurophysiology 98, 3423-3435.

Mott, D.D., and Dingledine, R. (2003). Interneuron Diversity series: Interneuron researchchallenges and strategies. Trends in neurosciences 26, 484-488.

Olsen, S.R., and Wilson, R.I. (2008). Lateral presynaptic inhibition mediates gain control in an olfactory circuit. Nature 452, 956-960.

Olshausen, B.A. (1996). Emergence of simple-cell receptive field properties by learning a sparse code for natural images. Nature 381, 607-609.

Ölveczky, B.P., Baccus, S.A., and Meister, M. (2003). Segregation of object and background motion in the retina. Nature 423, 401-408.

Otchy, T.M., Wolff, S.B., Rhee, J.Y., Pehlevan, C., Kawai, R., Kempf, A., Gobes, S.M., and Ölveczky, B.P. (2015). Acute off-target effects of neural circuit manipulations. Nature.

Paninski, L. (2003). Convergence properties of three spike-triggered analysis techniques. Network: Computation in Neural Systems 14, 437-464.

Park, I.M., and Pillow, J.W. (2011). Bayesian spike-triggered covariance analysis. In Advances in neural information processing systems, pp. 1692-1700.

Pillow, J.W., and Simoncelli, E.P. (2006). Dimensionality reduction in neural models: an information-theoretic generalization of spike-triggered average and covariance analysis. Journal of vision $6,9-9$.

Pitkow, X., and Meister, M. (2012). Decorrelation and efficient coding by retinal ganglion cells. Nature neuroscience 15, 628-635. 
Nonlinear effects of retinal amacrine cells

Rajan, K., and Bialek, W. (2013). Maximally informative "stimulus energies" in the analysis of neural responses to natural signals. PloS one 8, e71959.

Roska, B., Molnar, A., and Werblin, F.S. (2006). Parallel processing in retinal ganglion cells: how integration of space-time patterns of excitation and inhibition form the spiking output. Journal of Neurophysiology 95, 3810-3822.

Roska, B., and Werblin, F. (2003). Rapid global shifts in natural scenes block spiking in specific ganglion cell types. Nature neuroscience 6, 600-608.

Rust, N.C., Schwartz, O., Movshon, J.A., and Simoncelli, E.P. (2005). Spatiotemporal elements of macaque v1 receptive fields. Neuron 46, 945-956.

Sanes, J.R., and Masland, R.H. (2015). The types of retinal ganglion cells: current status and implications for neuronal classification. Annual review of neuroscience 38, 221-246.

Sceniak, M.P., Ringach, D.L., Hawken, M.J., and Shapley, R. (1999). Contrast's effect on spatial summation by macaque V1 neurons. Nature neuroscience 2, 733-739.

Schneidman, E., Berry, M.J., Segev, R., and Bialek, W. (2006). Weak pairwise correlations imply strongly correlated network states in a neural population. Nature 440, 1007-1012.

Schnitzer, M.J., and Meister, M. (2003). Multineuronal firing patterns in the signal from eye to brain. Neuron 37, 499-511.

Schwartz, O., Chichilnisky, E., and Simoncelli, E.P. (2002). Characterizing neural gain control using spike-triggered covariance. In Advances in neural information processing systems, pp. 269-276.

Schwartz, O., Pillow, J.W., Rust, N.C., and Simoncelli, E.P. (2006). Spike-triggered neural characterization. Journal of Vision 6, 13-13.

Schwartz, O., and Simoncelli, E.P. (2001). Natural signal statistics and sensory gain control. Nature neuroscience 4, 819-825. 
Nonlinear effects of retinal amacrine cells

Shapley, R., and Enroth-Cugell, C. (1984). Visual adaptation and retinal gain controls. Progress in retinal research 3, 263-346.

Sharpee, T., Rust, N.C., and Bialek, W. (2004). Analyzing neural responses to natural signals: maximally informative dimensions. Neural computation 16, 223-250.

Shlens, J., Field, G.D., Gauthier, J.L., Grivich, M.I., Petrusca, D., Sher, A., Litke, A.M., and Chichilnisky, E. (2006). The structure of multi-neuron firing patterns in primate retina. The Journal of neuroscience 26, 8254-8266.

Simoncelli, E.P., and Olshausen, B.A. (2001). Natural image statistics and neural representation. Annual review of neuroscience 24, 1193-1216.

Simoncelli, E.P., Paninski, L., Pillow, J., and Schwartz, O. (2004). Characterization of neural responses with stochastic stimuli. The cognitive neurosciences 3, 327-338.

Sincich, L.C., Horton, J.C., and Sharpee, T.O. (2009). Preserving information in neural transmission. The Journal of Neuroscience 29, 6207-6216.

Slllito, A.M., Grieve, K.L., Jones, H.E., Cudeiro, J., and Davls, J. (1995). Visual cortical mechanisms detecting focal orientation discontinuities.

Smirnakis, S.M., Berry, M.J., Warland, D.K., Bialek, W., and Meister, M. (1997). Adaptation of retinal processing to image contrast and spatial scale. Nature $386,69-73$.

Spratling, M.W. (2012). Unsupervised learning of generative and discriminative weights encoding elementary image components in a predictive coding model of cortical function. Neural Computation 24, 60-103.

Tanabe, S., Haefner, R.M., and Cumming, B.G. (2011). Suppressive mechanisms in monkey V1 help to solve the stereo correspondence problem. The Journal of Neuroscience 31, 8295-8305. 
Nonlinear effects of retinal amacrine cells

Theunissen, F.E., David, S.V., Singh, N.C., Hsu, A., Vinje, W.E., and Gallant, J.L. (2001). Estimating spatio-temporal receptive fields of auditory and visual neurons from their responses to natural stimuli. Network: Computation in Neural Systems 12, 289-316.

Touryan, J., Felsen, G., and Dan, Y. (2005). Spatial structure of complex cell receptive fields measured with natural images. Neuron 45, 781-791.

Touryan, J., Lau, B., and Dan, Y. (2002). Isolation of relevant visual features from random stimuli for cortical complex cells. The Journal of neuroscience 22, 10811-10818.

Twer, T., and MacLeod, D.I. (2001). Optimal nonlinear codes for the perception of natural colours. Network: Computation in Neural Systems 12, 395-407.

van Hateren, H. (2005). A cellular and molecular model of response kinetics and adaptation in primate cones and horizontal cells. Journal of vision 5, 5-5.

van Hateren, J.H., and van der Schaaf, A. (1998). Independent component filters of natural images compared with simple cells in primary visual cortex. Proceedings of the Royal Society of London B: Biological Sciences 265, 359-366.

Van Steveninck, R.D.R., and Bialek, W. (1988). Real-time performance of a movement-sensitive neuron in the blowfly visual system: coding and information transfer in short spike sequences. Proceedings of the Royal Society of London B: Biological Sciences 234, 379-414.

Werblin, F.S. (2011). The retinal hypercircuit: a repeating synaptic interactive motif underlying visual function. The Journal of physiology 589, 3691-3702.

y Arcas, B.A., and Fairhall, A.L. (2003). What causes a neuron to spike? Neural Computation $15,1789-1807$.

y Arcas, B.A., Fairhall, A.L., and Bialek, W. (2003). Computation in a single neuron: Hodgkin and Huxley revisited. Neural Computation 15, 1715-1749. 


\section{FIGURE 1}

A

visual
stimulus
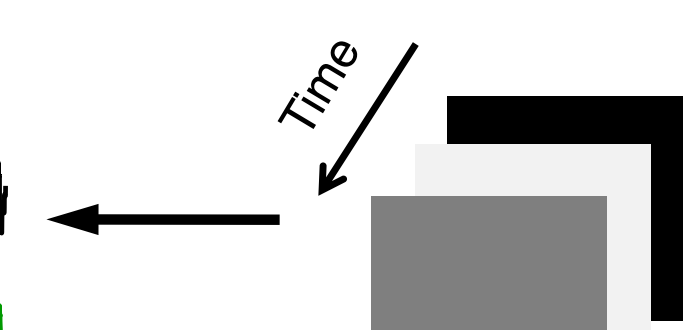

amacrine cell membrane voltage

current

stimulus

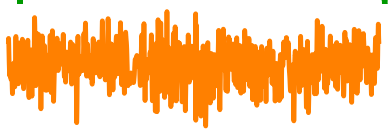

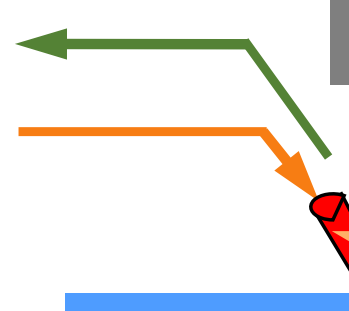

2s

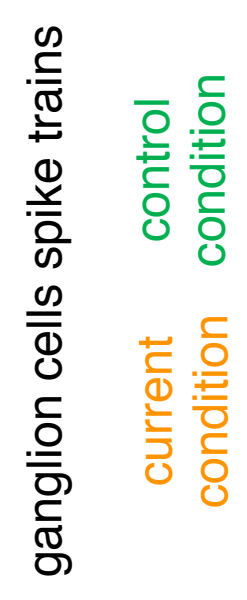

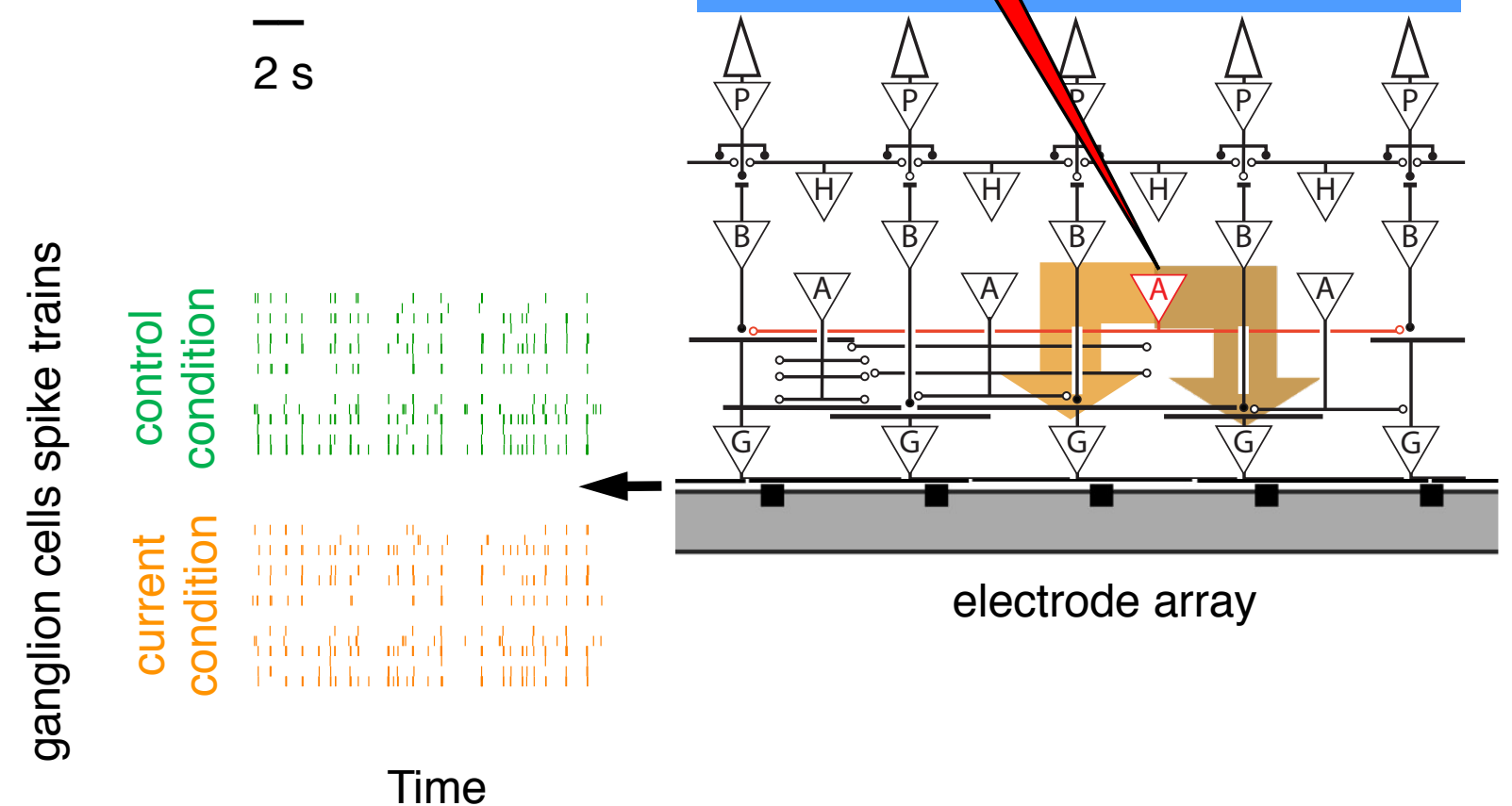

B
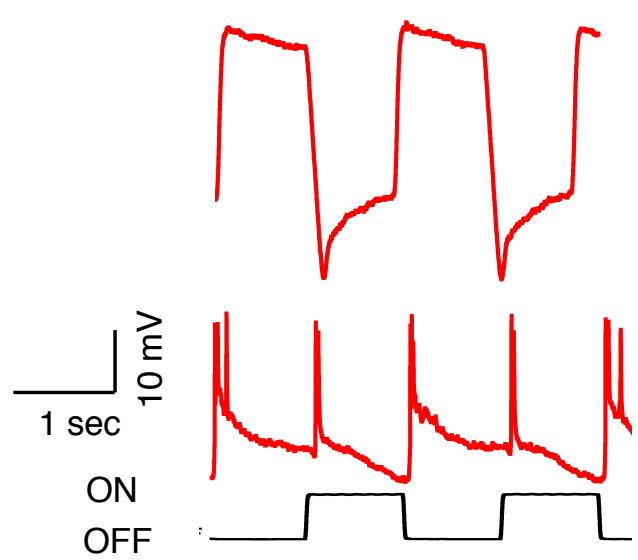

C

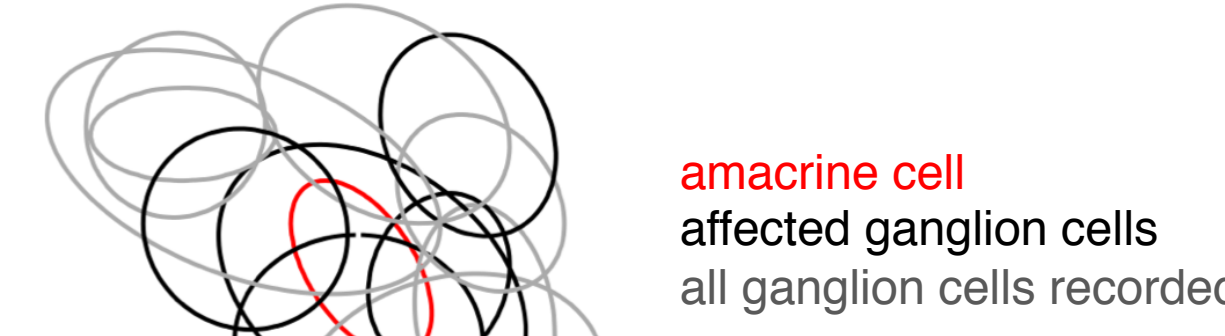

$120 \mu \mathrm{m}$

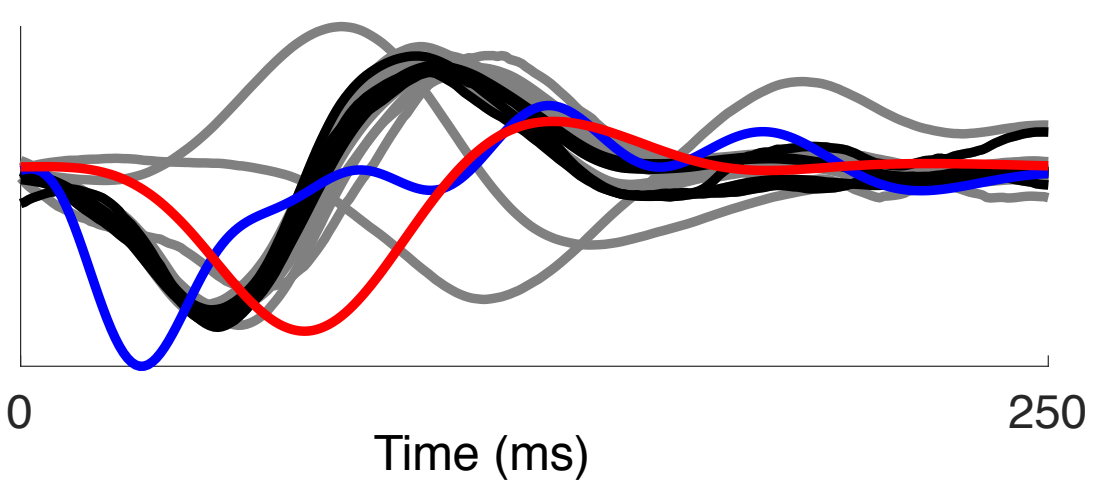


A
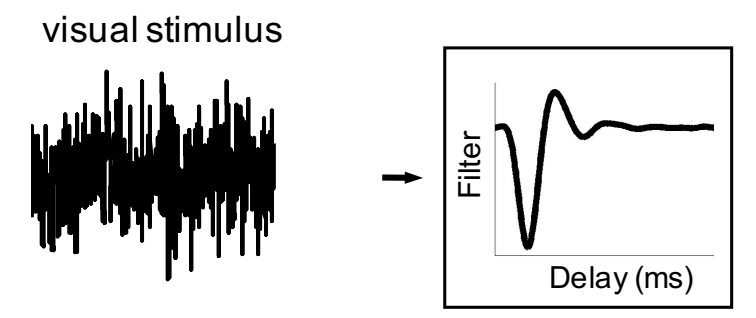

B current stimulus

C

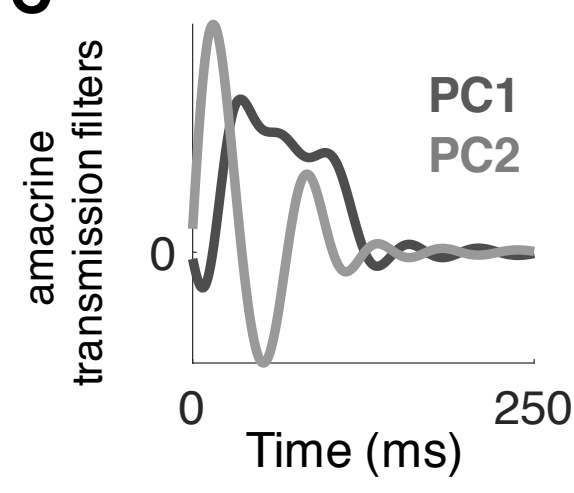

D

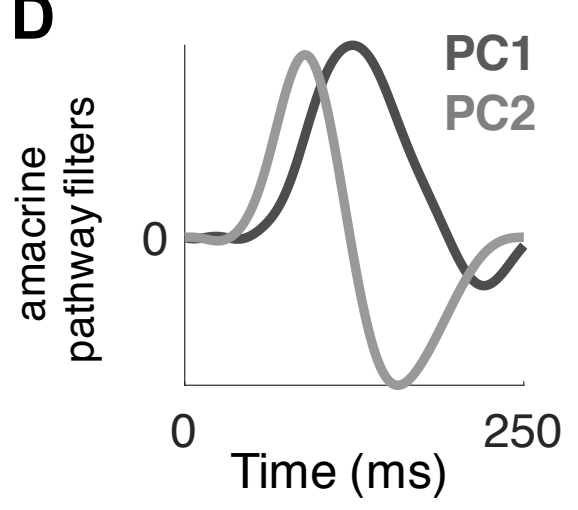

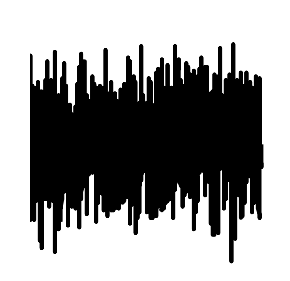

$\longrightarrow$ time
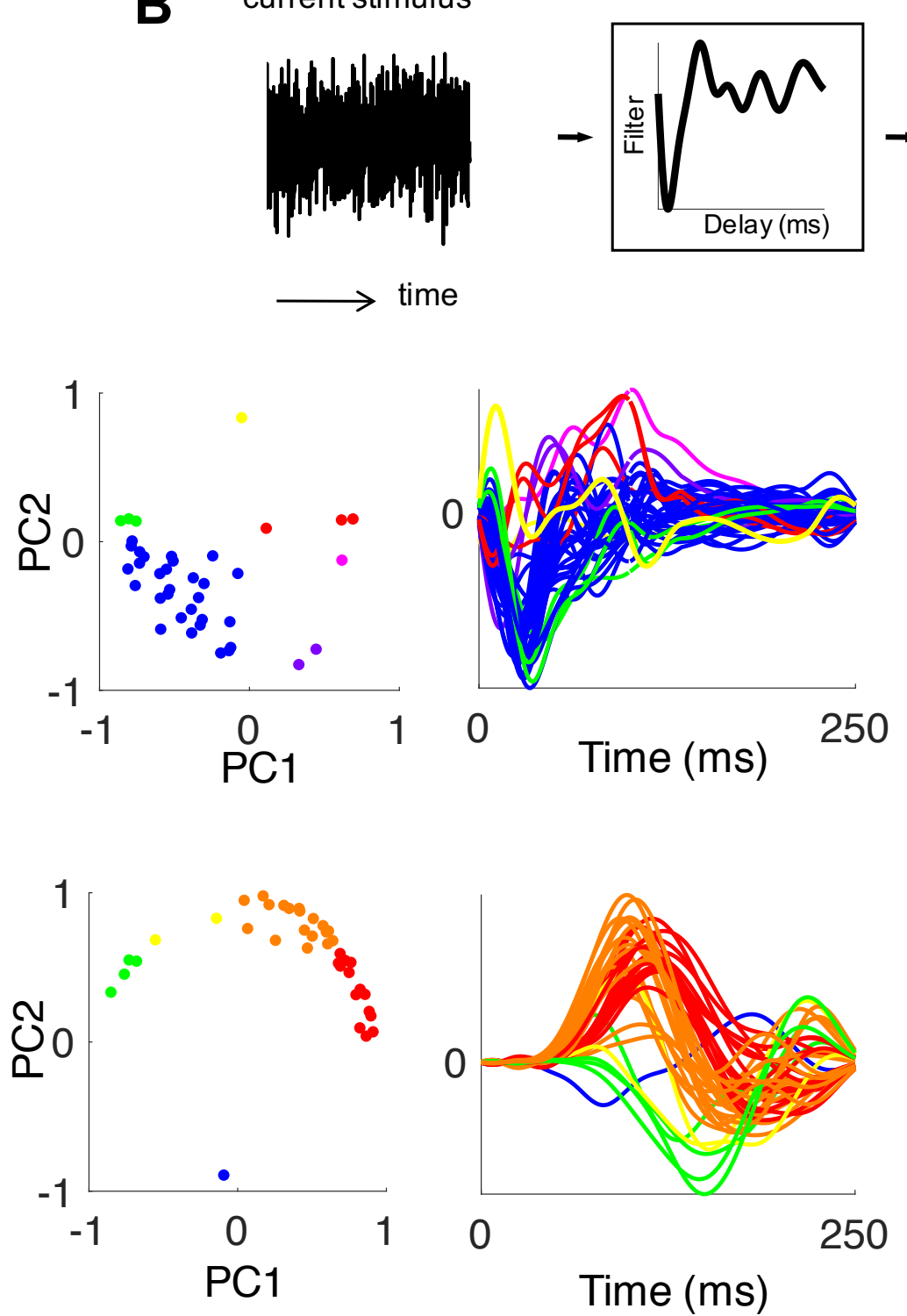

FIGURE 2

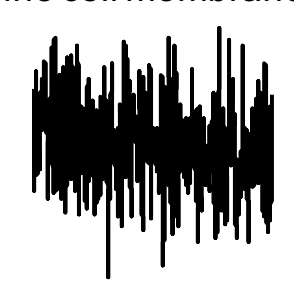

ganglion cell firing rate

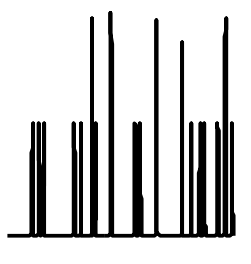

E
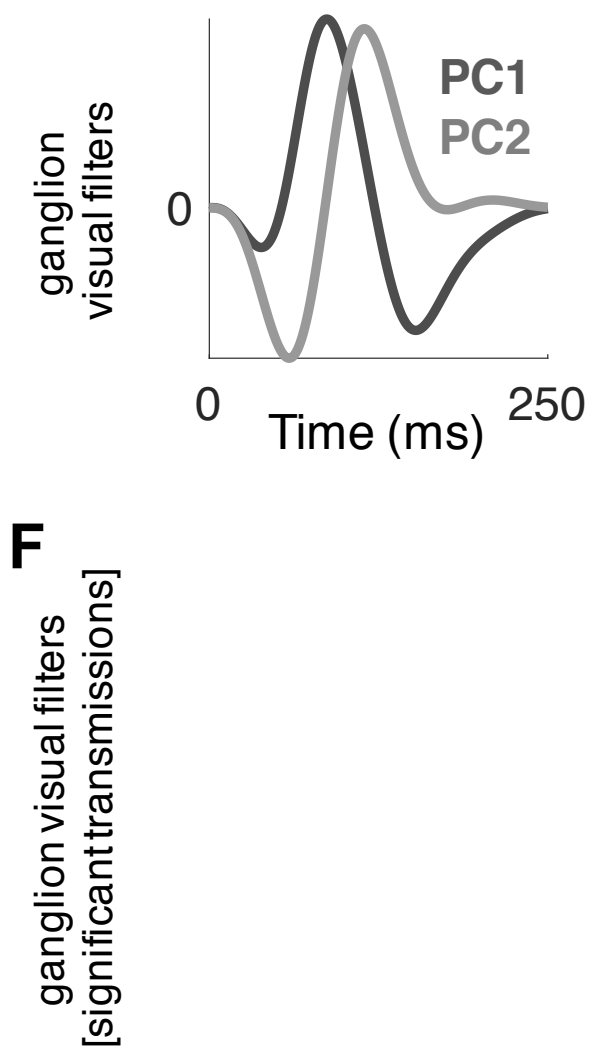
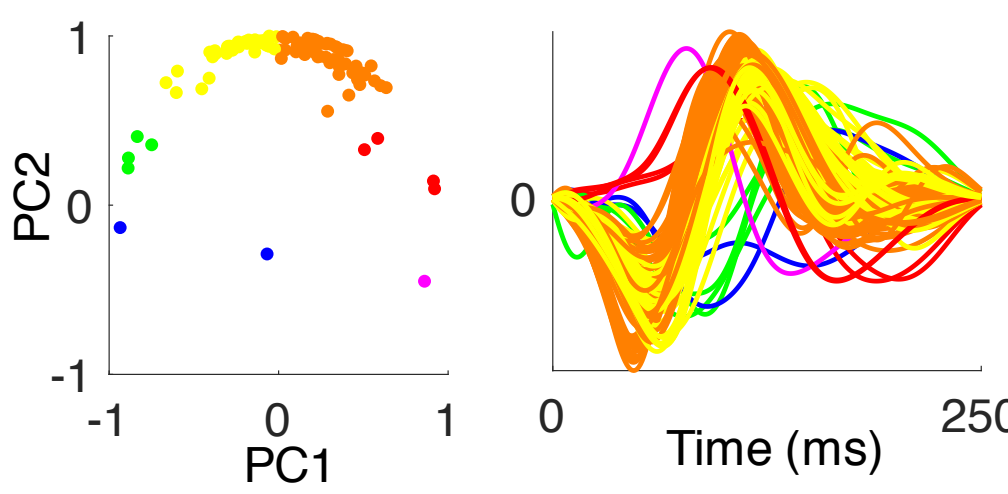

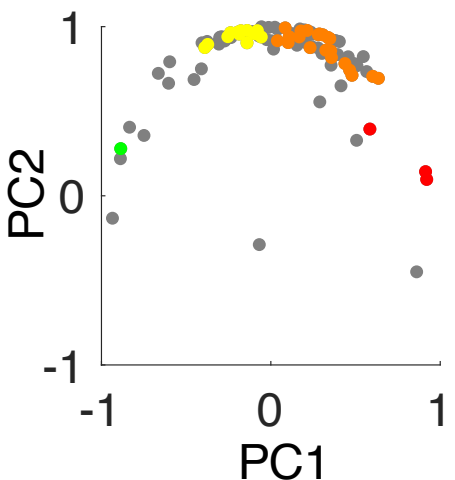


A
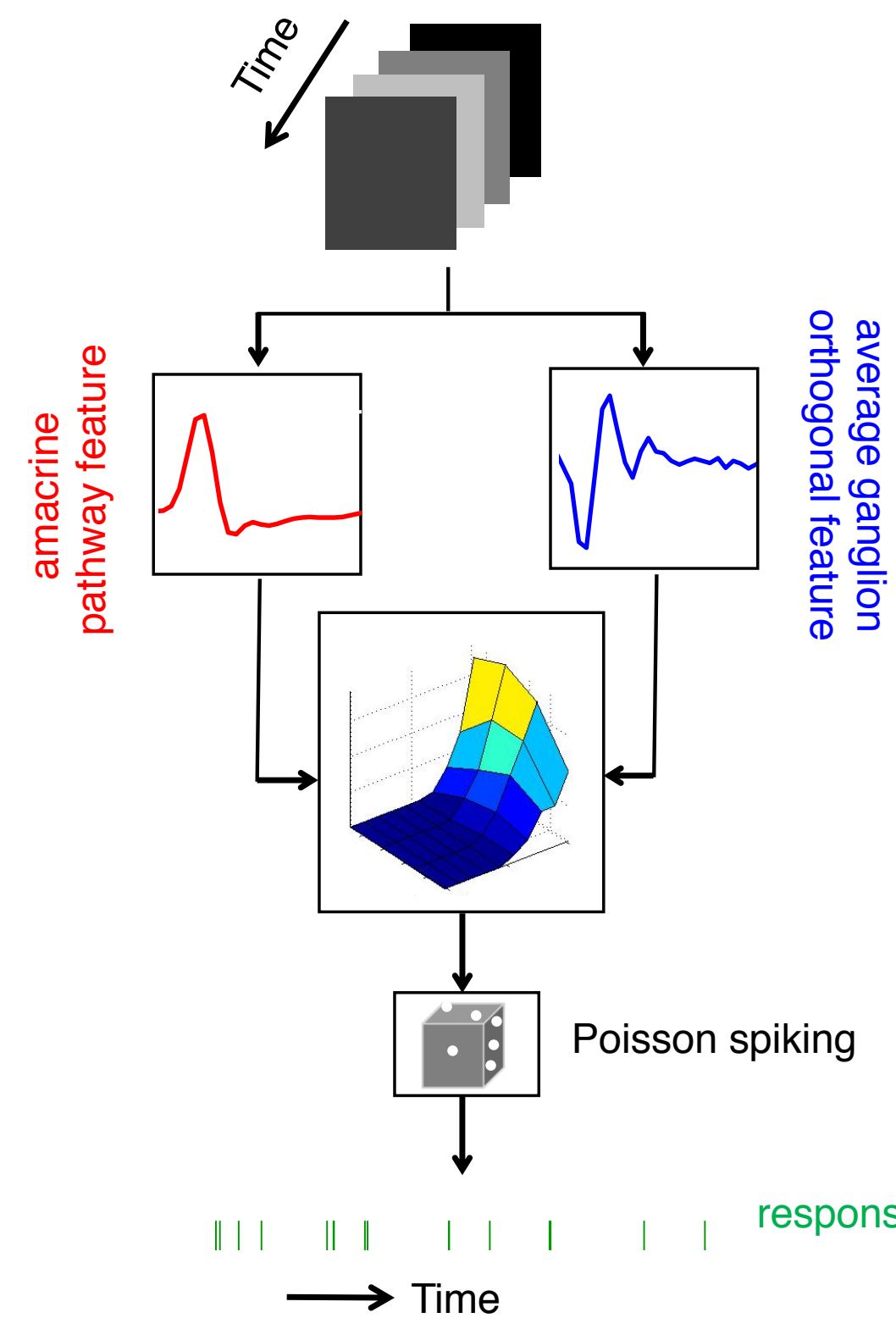

B

FIGURE 4

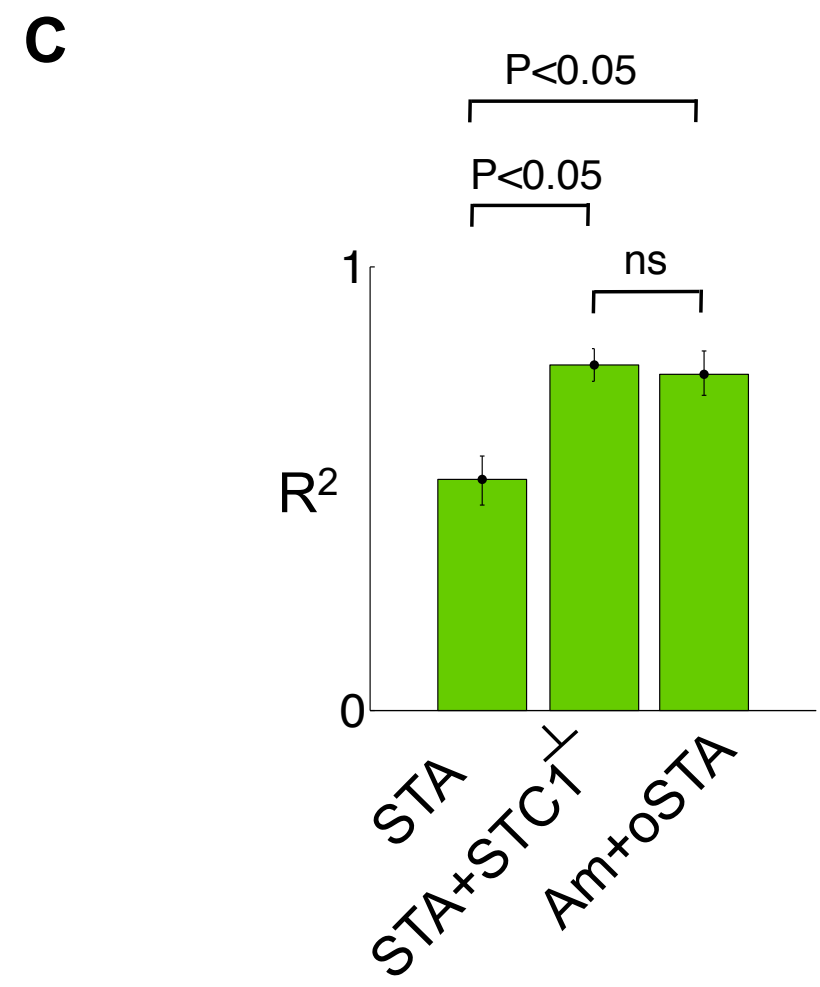




\section{A horizontally vertically}
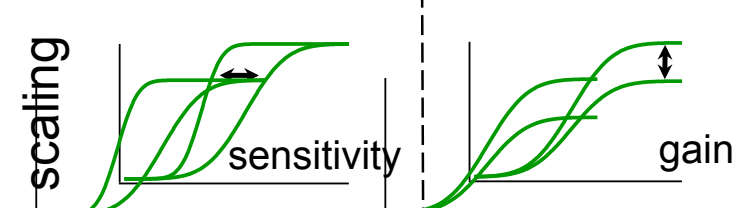

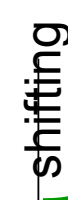
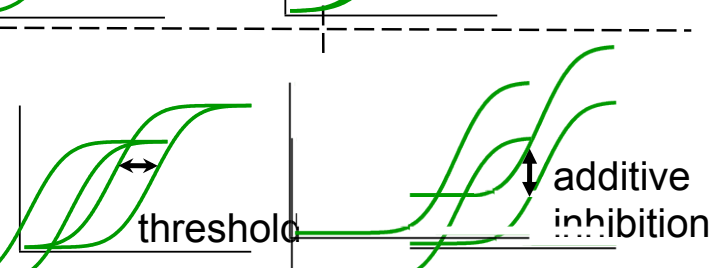

polarity reversal

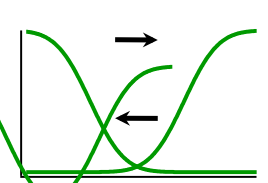

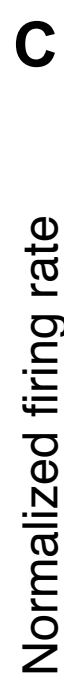

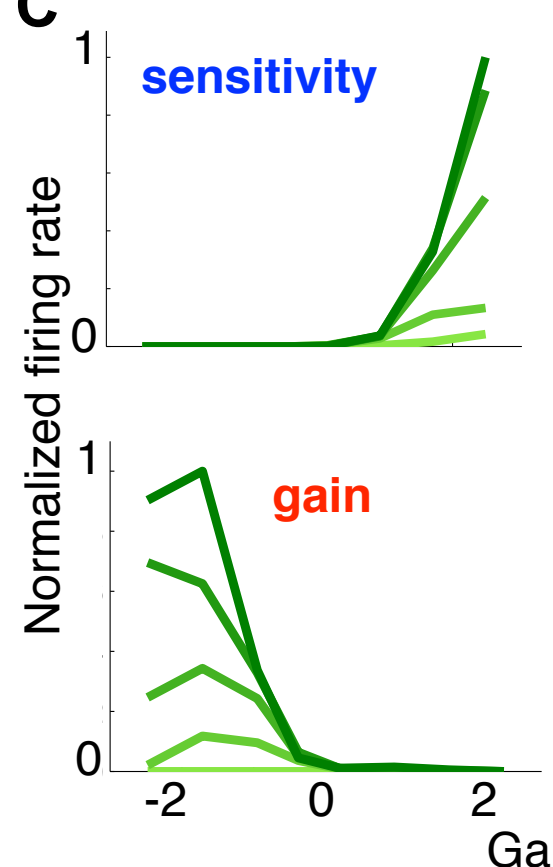

B

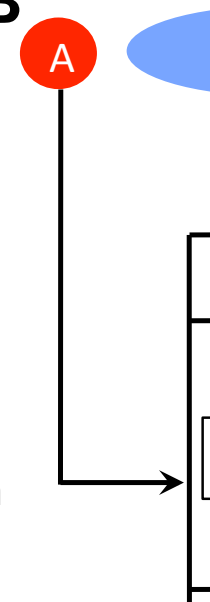

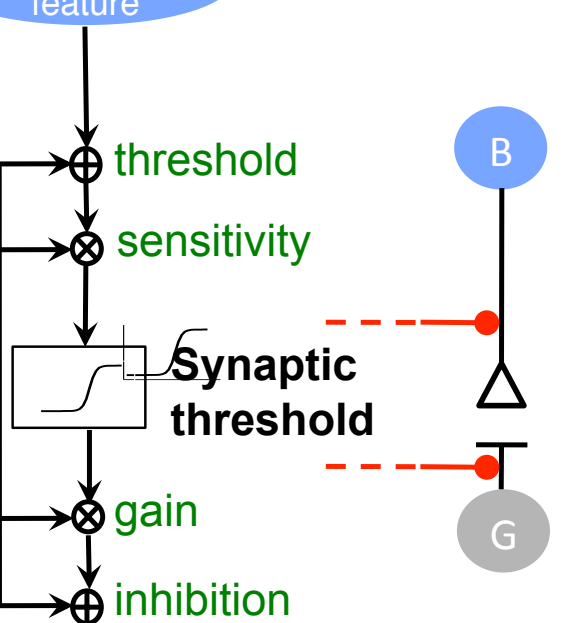

sensitivity

additive inhibition

polarity reversal

threshold \& gain

threshold A3-G2

gain
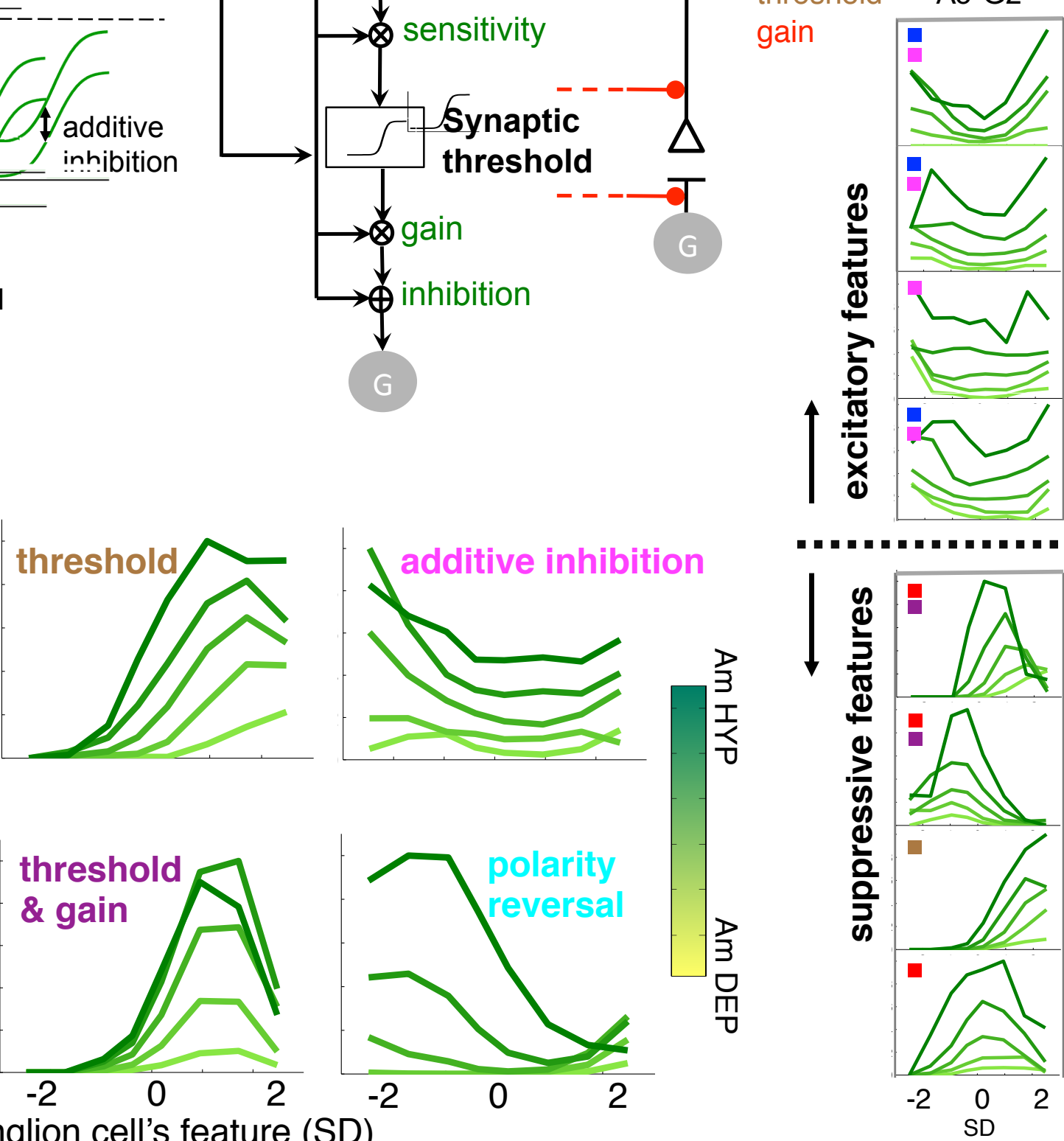

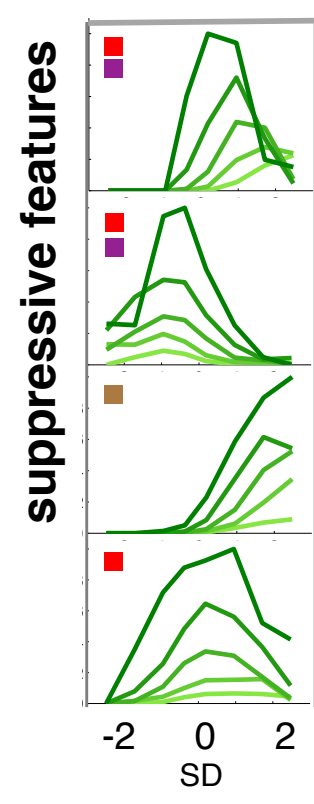

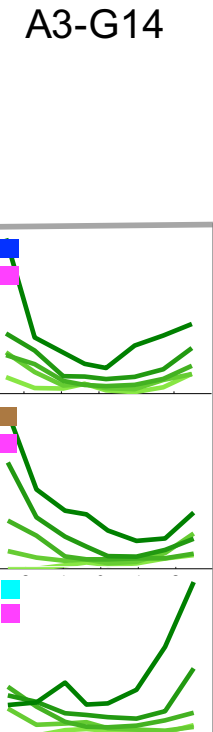

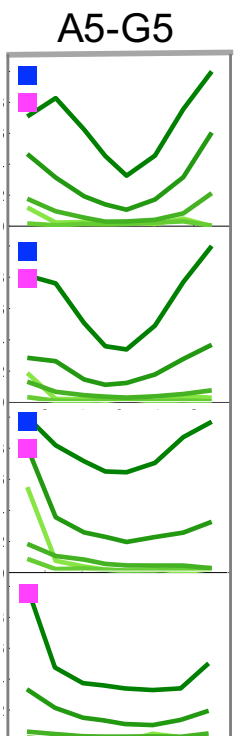

A8-G2

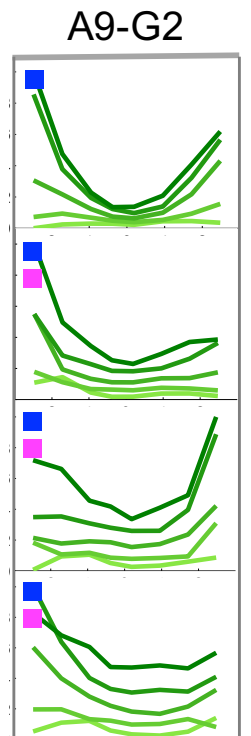

A9-G16
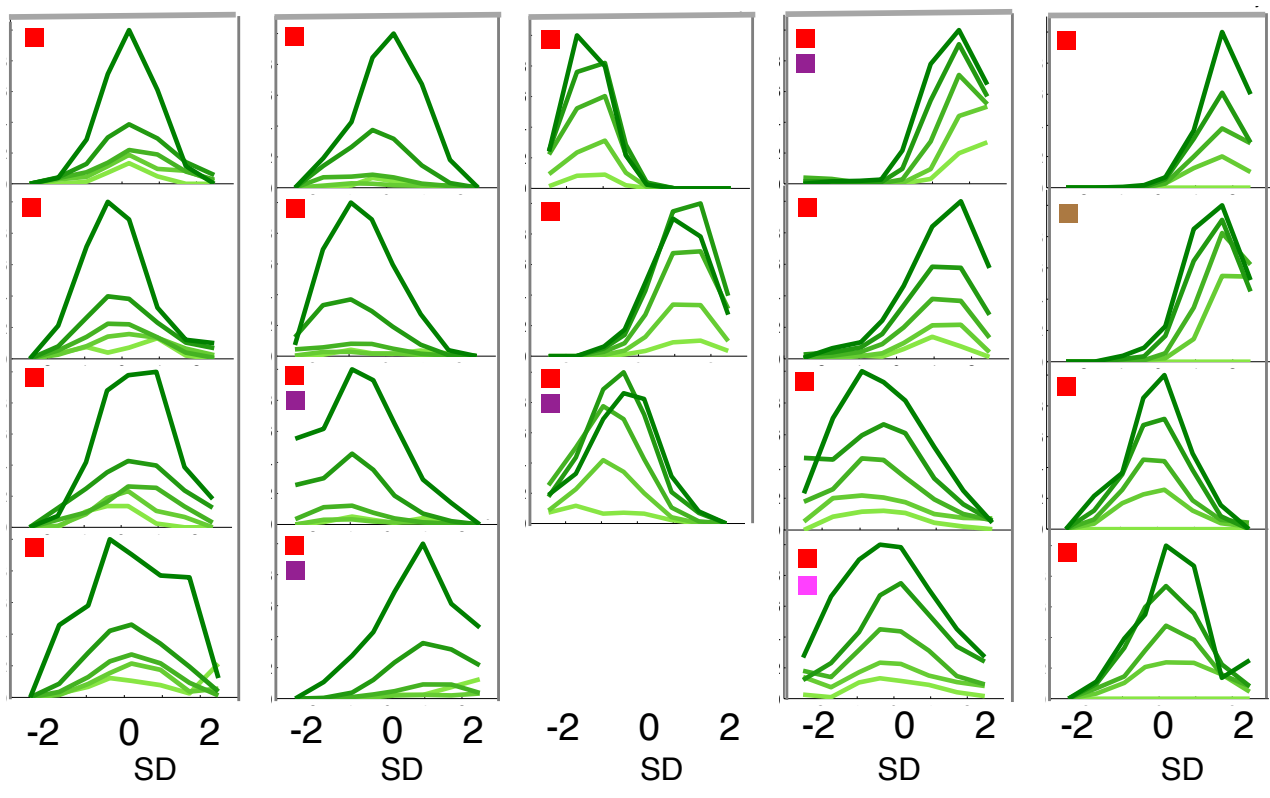

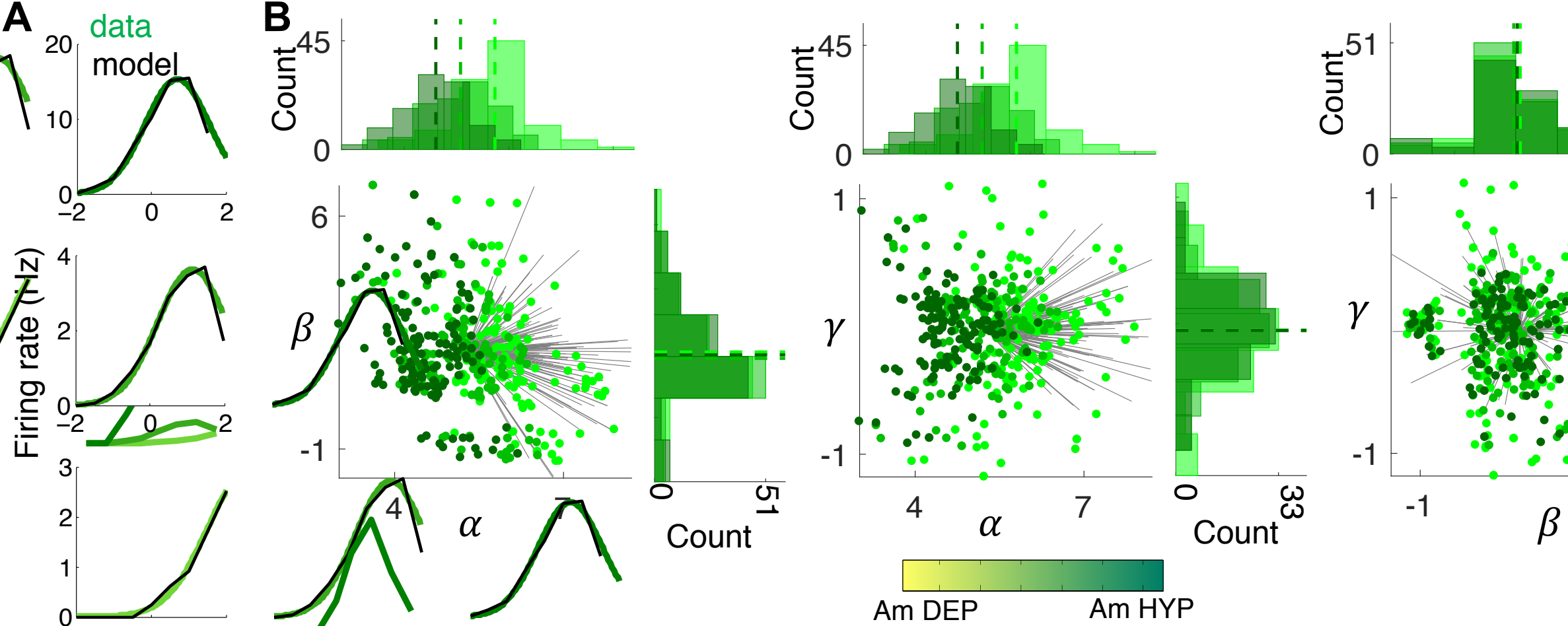

FIGURE 7

C
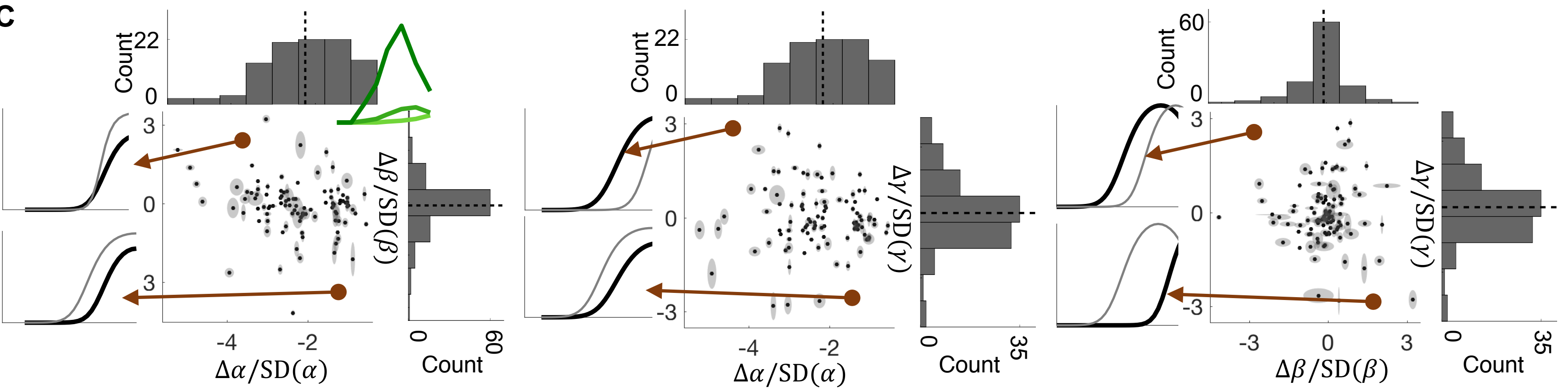


\section{FIGURE S1 (related to figure 2)}
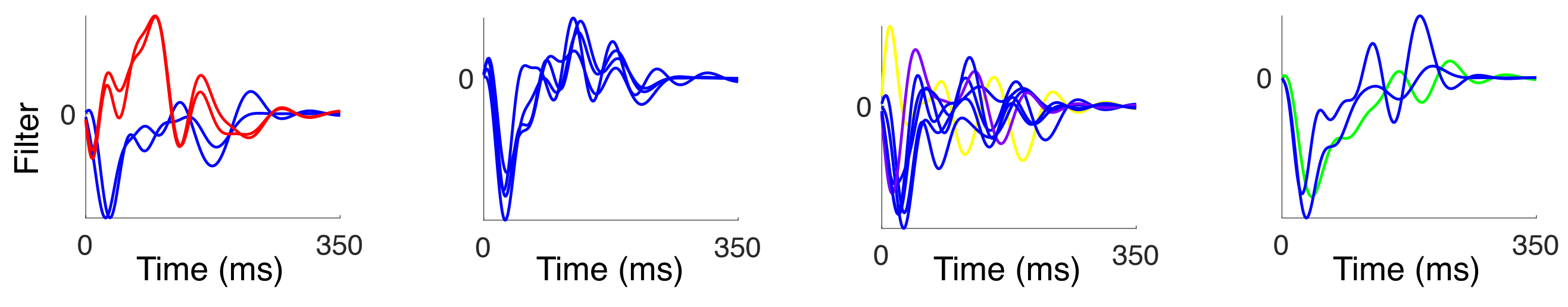


$\begin{array}{ccc}\text { ganglion } & \text { amacrine } & \text { firing rate } \\ \text { visual filters } & \text { pathway filters } & \text { nonlinearities }\end{array}$

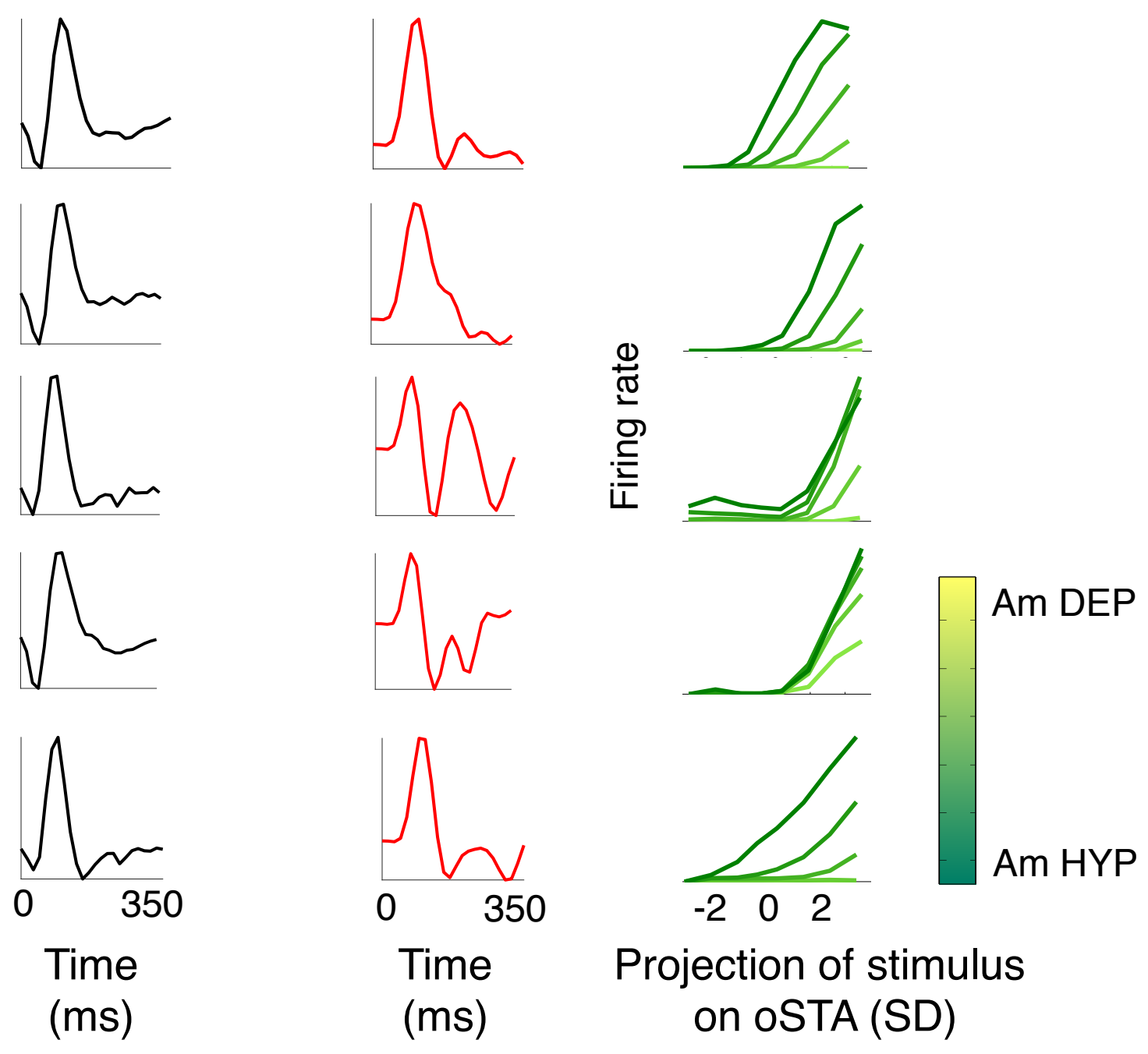


A

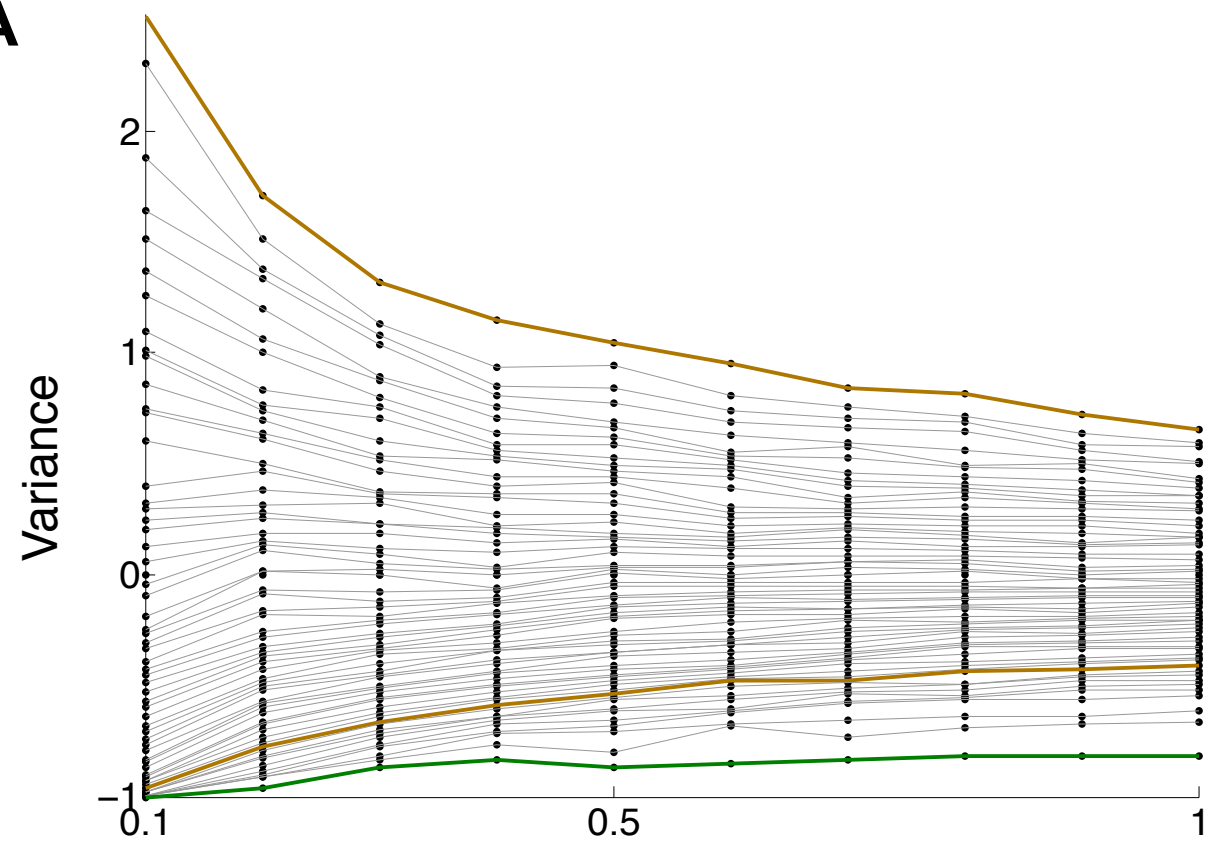

Fraction of spikes
B

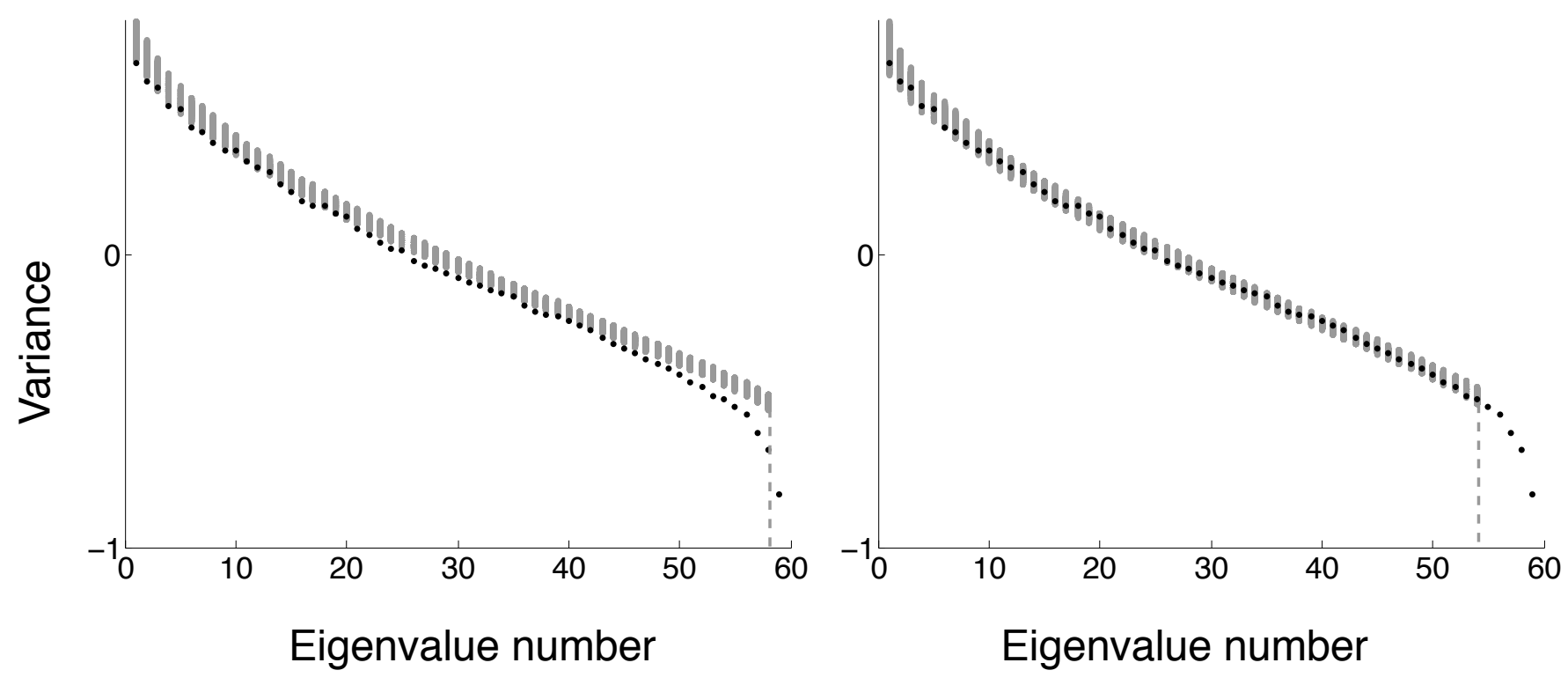

